ARTICLE

\title{
Lima1 mediates the pluripotency control of membrane dynamics and cellular metabolism
}

\author{
Binyamin Duethorn', Fabian Groll2 ${ }^{2}$, Bettina Rieger ${ }^{3}$, Hannes C. A. Drexler (1) ${ }^{4}$, Heike Brinkmann?, \\ Ludmila Kremer $^{5}$, Martin Stehling ${ }^{6}$, Marie-Theres Borowski (10 ${ }^{3}$, Karina Mildner ${ }^{7}$, Dagmar Zeuschner (i) ${ }^{7}$, \\ Magdalena Zernicka-Goetz (i) ${ }^{8,9}$, Marc P. Stemmler (10 ${ }^{10}$, Karin B. Busch ${ }^{3}$, Juan M. Vaquerizas (i) ${ }^{2,11,12}$ \& \\ Ivan Bedzhov (10 ${ }^{1 \times}$
}

Lima1 is an extensively studied prognostic marker of malignancy and is also considered to be a tumour suppressor, but its role in a developmental context of non-transformed cells is poorly understood. Here, we characterise the expression pattern and examined the function of Lima1 in mouse embryos and pluripotent stem cell lines. We identify that Lima1 expression is controlled by the naïve pluripotency circuit and is required for the suppression of membrane blebbing, as well as for proper mitochondrial energetics in embryonic stem cells. Moreover, forcing Lima1 expression enables primed mouse and human pluripotent stem cells to be incorporated into murine pre-implantation embryos. Thus, Lima1 is a key effector molecule that mediates the pluripotency control of membrane dynamics and cellular metabolism.

\footnotetext{
${ }^{1}$ Embryonic Self-Organization research group, Max Planck Institute for Molecular Biomedicine, Röntgenstraße 20, 48149 Münster, Germany. ${ }^{2}$ Regulatory Genomics group, Max Planck Institute for Molecular Biomedicine, Röntgenstraße 20, 48149 Münster, Germany. ${ }^{3}$ Institut für Integrative Zellbiologie und Physiologie, University of Münster, Schlossplatz 5, 48149 Münster, Germany. ${ }^{4}$ Mass Spectrometry Unit, Max Planck Institute for Molecular Biomedicine, Röntgenstraße 20, 48149 Münster, Germany. ${ }^{5}$ Transgenic Facility, Max Planck Institute for Molecular Biomedicine, Röntgenstraße 20, 48149 Münster, Germany. ${ }^{6}$ Flow Cytometry Unit, Max Planck Institute for Molecular Biomedicine, Röntgenstraße 20, 48149 Münster, Germany. ${ }^{7}$ Electron Microscopy Facility, Max Planck Institute for Molecular Biomedicine, Röntgenstraße 20, 48149 Münster, Germany. ${ }^{8}$ Mammalian Embryo and Stem Cell Group, Department of Physiology, Development, and Neuroscience, University of Cambridge, Downing Street, Cambridge CB2 3EG, UK. ${ }^{9}$ Plasticity and SelfOrganization Group, Division of Biology and Biological Engineering, California Institute of Technology (Caltech), Pasadena, CA 91125, USA. ${ }^{10}$ Department of Experimental Medicine 1, Nikolaus-Fiebiger-Center for Molecular Medicine, FAU University Erlangen-Nürnberg, Erlangen, Germany. ${ }^{11}$ MRC London Institute of Medical Sciences, Du Cane Road, W12 ONN London, UK. ${ }^{12}$ Institute of Clinical Sciences, Faculty of Medicine, Imperial College London, Hammersmith Hospital Campus, Du Cane Road, London W12 ONN, UK.凶email: ivan.bedzhov@mpi-muenster.mpg.de
} 
$\mathrm{T}$ he most frequent types of cancer originate from epithelial cells, which aberrantly activate signalling cascades that promote cell proliferation and allow cells to evade apoptosis ${ }^{1}$. The cancer cells often reprogramme their energy metabolism, lose epithelial morphology and activate invasion and metastasis, enabling a benign adenoma to transform into an invasive carcinoma, which correlates with poor patient prognosis. The malignant transformation is associated with reduced intercellular adhesion as well as cytoskeletal and signalling reorganisation, in a process known as epithelial-to-mesenchymal transition $(\mathrm{EMT})^{2}$.

The process of EMT as well as the activation of several signalling pathways, such as Wnt, Notch and Hedgehog, are common features in both embryonic development and cancer ${ }^{3}$. The EMT, which mediates E-cadherin (E-cad) downregulation, is the driving force enabling malignancy and is also a key step in the process of gastrulation ${ }^{1}$. Similarly, the canonical Wnt $/ \beta$-catenin $(\beta$-cat) signalling is essential for the patterning of the early mouse embryo $^{4,5}$. However, aberrant activation of this pathway can initiate tumorigenesis by promoting the expression of downstream oncogenes ${ }^{6}$.

E-cad and $\beta$-cat, together with several actin-binding proteins, such as $\alpha$-catenin ( $\alpha$-cat) and Limal (also known as EPLIN; epithelial protein lost in neoplasm), form the adherens junction (AJ) complex ${ }^{1,7}$. The role of the main components of this complex has been extensively studied in the context of both cancer and embryogenesis, which substantially widens our understanding of their expression pattern, functions and regulation. Yet, it is so far unknown how Lima1, which is considered to be a prognostic marker and tumour suppressor ${ }^{8}$, functions during embryonic development.

From previous studies, the Limal locus is known to harbour two promoter regions, which drive the expression of the shorter Lima1- $\alpha$ (600 amino acids) and the longer Lima1- $\beta$ (759 amino acids) isoforms. Both isoforms contain two actin-binding domains and a central LIM domain ${ }^{9,10}$. Recombinant Lima1- $\alpha$ and $-\beta$ proteins suppress the depolymerization of actin filaments and cross-link the filaments in bundles ${ }^{11}$. In epithelial cells, the actin fibres are linked to the cadherin-catenin complex (CCC) via $\alpha$-cat on the $\mathrm{AJ}^{12}$. Limal can bind $\alpha$-cat and F-actin, thereby mediating the contact of the CCC with the cytoskeleton ${ }^{7}$ and acting as a mechanosensitive regulator ${ }^{13}$.

In the context of cancer, Limal downregulation is implicated in the progression of oral, prostate and breast cancer ${ }^{10,14-17}$. In prostate cancer cells, Lima1 depletion promotes AJ disassembly and cell invasion ${ }^{18}$; conversely, in oesophageal and breast cancer cells, Lima1- $\alpha$ overexpression decreases tumour growth and invasiveness, acting as a tumour suppressor ${ }^{16,17}$. Moreover, Limal function is also involved in the cellular metabolism, as shown in the gut, where Limal binds to intracellular cholesterol transporters and facilitates cholesterol absorption ${ }^{19}$.

As the majority of studies examining Limal have been performed in pathological situations, the role of Limal in a developmental context of non-transformed cells is still poorly understood. Therefore, we used the mouse embryo and embryoderived stem cell lines to analyse the expression pattern and function of Limal.

Here, we show that Limal expression was not confined only to the epithelial tissues of the foetus. During the pre-implantation development, Limal was initially enriched in the polar body and later in the pluripotent inner cell mass (ICM) of the early embryo. We determined that Limal is under the transcriptional control of the naïve pluripotency circuit and is required for suppressing the formation of membrane blebs in mouse and human pluripotent stem cells. In addition, we found that Limal is required for proper mitochondrial function in a cell-autonomous manner and is essential for the growth of solid tumours. Strikingly, ectopic expression of Limal in human pluripotent stem cells enabled these cells to be incorporated into murine pre-implantation embryos. Thus, Limal is a key effector molecule mediating pluripotency control of membrane dynamics and cellular metabolism.

\section{Results}

Limal expression pattern during mouse embryonic development. The process of embryogenesis starts from a single cell (zygote), which gives rise to both the embryonic and extraembryonic lineages of the early embryo. After implantation, a complex cascade of cell fate decisions and morphogenetic events form the different tissues and organs of the developing embryo. To analyse Limal expression during murine development, we isolated E14.5 embryos, which were sectioned and stained for Limal. Alongside this, we examined the localisation of the major components of the CCC, namely E-cad, $\alpha$-E-catenin ( $\alpha$-E-cat), $\mathrm{N}$-cadherin $(\mathrm{N}$-cad), $\alpha-\mathrm{N}$-catenin $(\alpha-\mathrm{N}$-cat) and $\beta$-cat. In E14.5 embryos, E-cad and $\alpha$-E-cat were expressed mainly in epithelial compartments, such as the inner lining of the stomach (Fig. 1a, blue arrowhead). N-cad and $\alpha-\mathrm{N}$-cat were detected in the muscle fibres of the stomach wall (Fig. 1a, magenta arrowhead) as well as in the nervous system, whereas various levels of $\beta$-cat were broadly detectable throughout the embryo (Fig. 1a and S1). Although Limal is considered to be an epithelial protein ${ }^{10}$, unexpectedly its expression was not confined only to epithelial tissues. Similar to $\beta$-cat, Lima1 was broadly expressed, occupying both E-cad and N-cad expression domains (Fig. 1a and S1), suggesting that the potential functions of Lima1 in the developing embryo may span beyond epithelial homoeostasis.

Next, we analysed the expression of Limal during the preimplantation embryogenesis. Using available transcriptomics data ${ }^{20}$, we found that Limal mRNA is present in GV and MII oocytes as maternal transcripts, and later the zygotic expression exhibits a sharp increase after the 16-cell stage (Fig. 1b). Staining GV and MII oocytes for Limal revealed that the protein is localised in the cytoplasm and the cortex, with strong enrichment in the cortical cup overlying the spindle of the MII oocytes (Fig. 1c). Interestingly, in the zygote, Limal accumulated in the extruded polar body, where it remained detectable during the next cleavage stages. Later, Lima1 expression appeared stronger in the inside cells of the 16-cell morula and the ICM of the early blastocyst (Fig. 1c). To validate these observations, we examined the Limal expression pattern in combination with lineage markers, discriminating the inner/outer and the ICM/trophectoderm (TE) cells of morula and early blastocyst stage embryos, respectively. In comparison to E-cad, $\beta$-cat and $\alpha$-E-cat, which were overall ubiquitously expressed in both compartments, Lima1 was enriched in the Sox $2+/ \mathrm{Cdx} 2-$ inner cells of the preimplantation embryo (Fig. 1d, e).

At mid and late blastocyst stage, we found that the Sox17+ primitive endoderm (PE) cells toned-down Limal expression (Fig. 1f, arrowhead), whereas the pre-implantation epiblast (Sox17-/Cdx2-) maintained a relatively high level of Lima1. In addition, Limal also became detectable in the TE (Fig. 1f). Conversely, after implantation at early egg cylinder stage (E5.5), Limal expression was decreased in the extraembryonic ectoderm (ExE) and the post-implantation epiblast (Fig. 1f). Thus, Limal exhibited a dynamic expression pattern in the embryonic and extraembryonic lineages during early mouse development. In the next experiments we focused on examining Limal function in the pluripotent lineage. 
a
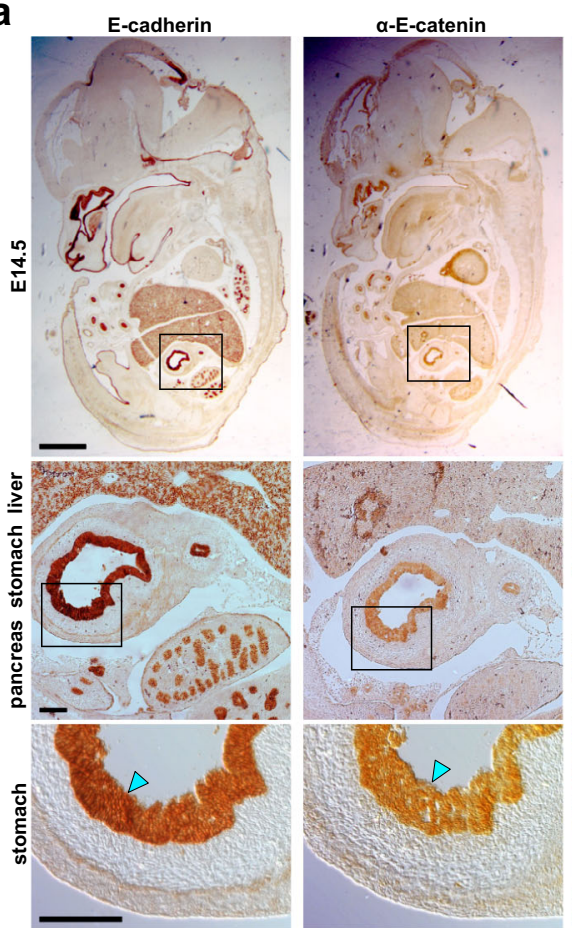

b

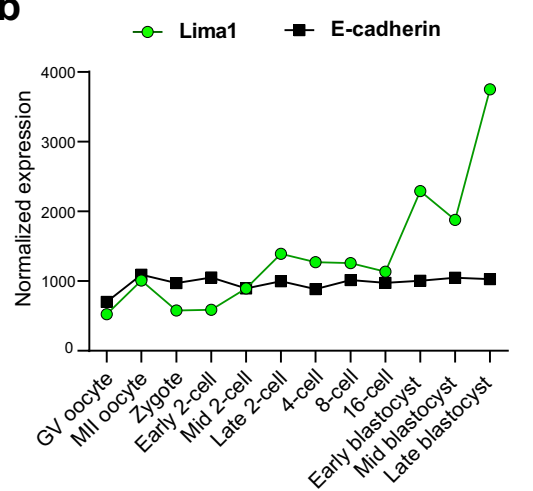

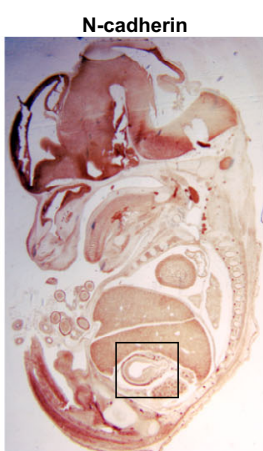
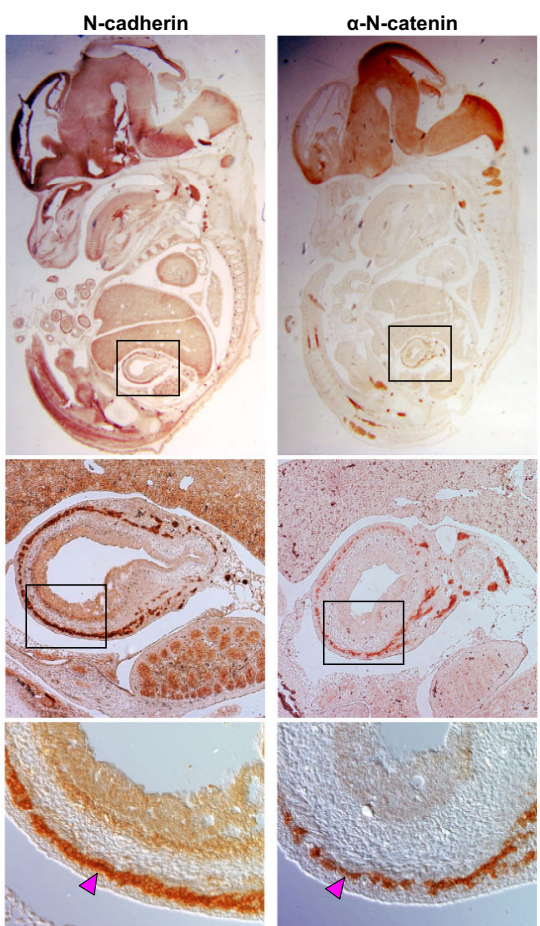

C

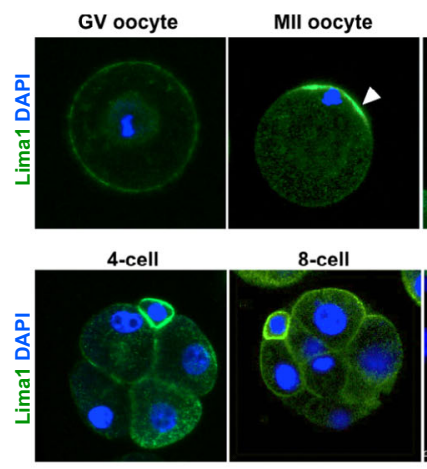

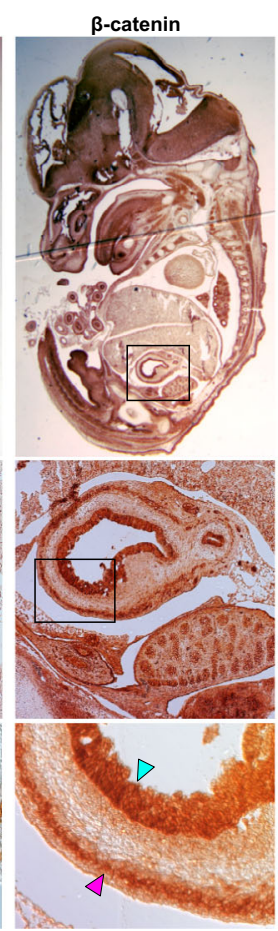
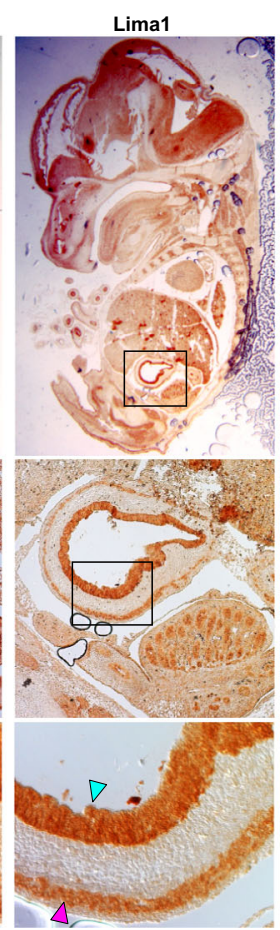

d

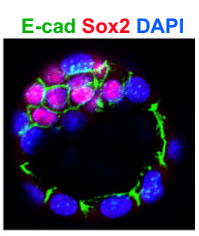

Lima1 Sox2 DAPI

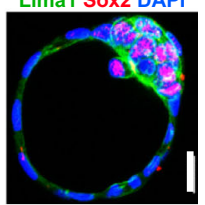

e

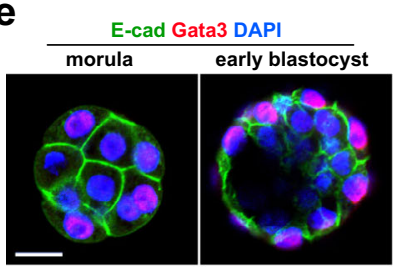

$\alpha-E-c a t$ Gata3 DAPI

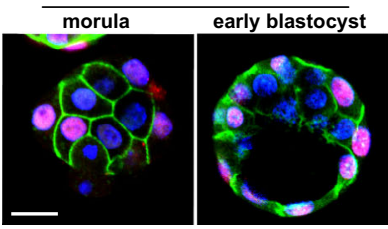

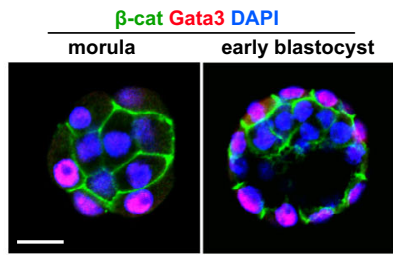

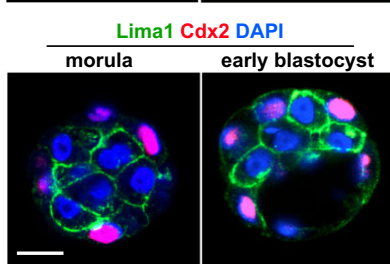

$\mathbf{f}$

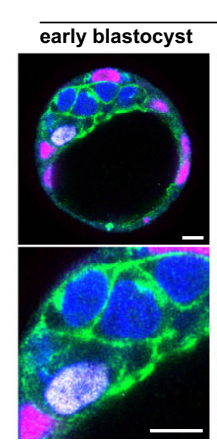

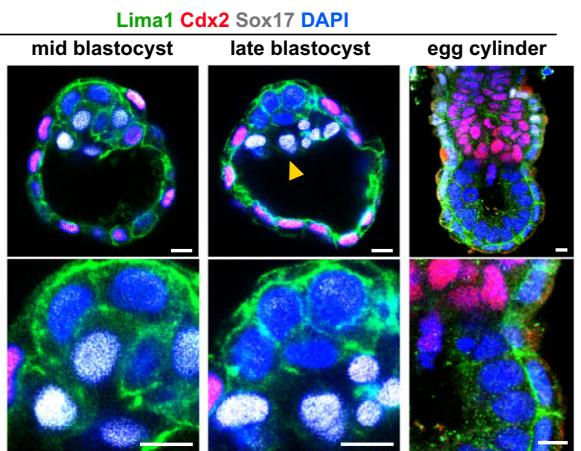

Fig. 1 Lima1 expression pattern during mouse embryonic development. a E14.5 embryos sectioned and stained for E-cad, $\alpha$-E-cat, $N$-cad, $\alpha$-N-cat, $\beta$-cat or Lima1. Blue and magenta arrows indicate epithelial and muscle layers, respectively. b Normalized expression levels of Lima1 and E-cad during mouse preimplantation development, based on Wang et al., 2004. c Oocytes and pre-implantation embryos stained for Lima1. Arrowheads indicate Lima1 localisation on the cortical cap in MII oocyte and in the polar body at the zygote stage. d Blastocyst stage embryos stained for Sox2 and E-cad or Sox2 and Lima1. e Morula and blastocyst stage embryos stained for AJ components, Lima1 and TE lineage markers (Gata3 or Cdx2). f Blastocyst and egg cylinder stage embryo stained for Lima1, Cdx2, Sox17 and DAPI. The PE is indicated by an arrowhead. Scale bars, (a), $1000 \mu \mathrm{m}$, upper panel and $100 \mu \mathrm{m}$, lower panels; (c), (d) and (e), $20 \mu \mathrm{m} ;(\mathbf{f}), 10 \mu \mathrm{m}$. Experiments were repeated independently at least three times with similar results (a, c, d, e, f). Related to Figure S1. 
Limal expression is under the control of the naïve pluripotency circuit. As Limal was enriched in the pre-implantation epiblast, we asked whether its expression is maintained in vitro, in embryonic stem cells (ESC). Murine ESC are typically co-cultured with mitotically inactivated mouse embryonic fibroblasts (MEFs) in serum-containing medium, supplemented with leukaemia inhibitory factor (Lif) ${ }^{21}$; alternatively, ESC can be grown without MEFs in serum-free N2B27 medium, supplemented with Lif and $2 \mathrm{i}$ (Gsk3 and Mek inhibitors) ${ }^{22}$. In the $2 \mathrm{i} /$ Lif culture conditions, we found that the longer Lima1- $\beta$ isoform was predominantly expressed, whereas, in the presence of MEFs, both Lima1- $\alpha$ and $-\beta$ isoforms were detectable (Fig. 2a). However, MEFs themselves showed only Limal- $\alpha$ expression, indicating that Lima1- $\beta$ is the main isoform present in pluripotent cells (Fig. 2a).

The mouse blastocyst and egg cylinder stage embryos are also a source of trophoblast stem cells (TSC), which are derived from the trophoblast lineage ${ }^{23}$. As Limal expression was lower in the TE (early blastocyst) and ExE (egg cylinder), compared to the pre-implantation epiblast, we asked whether TSC and ESC recapitulate this expression pattern in vitro. Similar to the pre-implantation embryo, Limal was enriched in ESC but not TSC, whereas E-cad was ubiquitously expressed in both TSC and ESC (Fig. 2b and S2A). In addition, using a forced expression of $\mathrm{Cdx} 2^{24}$, we reprogrammed the ESC to TSClike cells (Fig. 2c) and found that, in contrast to the E-cad expression that remained unchanged, Limal was downregulated in the TSC-like cells (Fig. 2d-f).

As ESC capture features of the naïve pluripotent lineage of the blastocyst stage embryo ${ }^{25}$, we asked whether Lima1 is under the transcriptional control of the naïve pluripotency network. To test this, we compared Limal expression in naïve ESC to more developmentally advanced pluripotent stem cells. Briefly, we treated ESC with Fgf2/Activin, which causes cells to exit naïve pluripotency and transition into so-called epiblast-like cells (EpiLC) ${ }^{26}$ (Fig. 2g). These cells shut down the expression of naïve pluripotency transcription factors, such as Nanog and Esrrb (Fig. 2k and S2B), establishing an early post-implantation epiblast-like state ${ }^{26}$. Similar to Nanog and Esrrb, Limal expression was diminished in EpiLC, whereas E-cad and $\beta$-cat protein levels remained overall unchanged (Fig. $2 \mathrm{~h}, \mathrm{k}$ and S2B). In addition, Limal was also enriched in naïve human induced pluripotent stem cells (naïve hiPSC) in comparison to primed (conventional) hiPSC (Fig. S2C).

During the transition from the naïve to the primed state of pluripotency, Lima1 was substantially downregulated on the mRNA level, exhibiting similar transcriptional dynamics as the naïve pluripotency factor Nanog (Fig. 2l). Moreover, analysis of available ChIP-seq data on pluripotency transcription factor binding in $\mathrm{ESC}^{27-35}$ (Table S1) revealed peaks for Nanog, Nr0b1, Klf4, Sox2, Oct4, Sall4, $\beta$-cat and Tcf3 in the vicinity of the Lima1- $\beta$ promoter (Fig. $2 \mathrm{~m}$ ). This suggests that Limal is a potential target gene of the naïve pluripotency circuit and may act as a downstream effector, mediating naïve pluripotency control of cytoskeletal dynamics.

Limal controls the membrane dynamics in ESC. To analyse Lima1 functions, we established Limal knockout (ko) ESC using the CRISPR/Cas9 approach (Fig. 3a). We deleted the genomic region containing exons 4 and 5 to generate a premature stop codon, thus abolishing Limal translation (Fig. 3b). Morphologically, Lima1 KO ESC formed typical dome-shaped colonies, undistinguishable from the wild-type (WT) ESC. The main components of the CCC, namely E-cad, $\beta$-cat and $\alpha$-E-cat, were properly localised on the membrane, and Lima1 KO ESC did not exhibit intercellular adhesion defects (Fig. 3c).

To understand whether the loss of Limal can affect the status of undifferentiated stem cells, we performed RNA-seq analysis comparing WT and Lima1 KO ESC (Fig. 3d and S3A, Supplementary. Dataset 1). We found no substantial changes in the expression of core, naïve or primed pluripotency markers (Fig. 3e). In addition, Limal loss of function did not result in an upregulation of ecto-, endo- or mesodermal markers (Fig. S3B), showing that Limal depletion does not promote ESC differentiation.

Interestingly, gene ontology (GO) analysis revealed the enrichment of gene expression associated with epithelial and amoeboid-like cell migration as well as the downregulation of genes involved in cellular metabolism in Limal KO ESC. In addition, GO terms such as DNA replication, nuclear division and chromosome segregation were reduced, suggesting that Limal KO ESC may exhibit reduced rates of mitosis and potentially a decrease in cell proliferation (Fig. $3 \mathrm{f}$ and S3C-S3D). To further explore Limal functions, we characterised the Lima1 interactome using APEX2 (peroxidase proximity labelling with ascorbate peroxidase) assay ${ }^{36}$. We fused the Lima1- $\beta$ isoform (full length) at the C-terminus to an HA-tag, followed by the APEX2 coding sequence (Fig. $3 \mathrm{~g}, \mathrm{~h}$ ). In the presence of biotin-phenol (BP), a pulse of hydrogen peroxide results in the generation of biotin-phenoxyl radicals by APEX2, which covalently labels proximal endogenous proteins (Fig. $3 \mathrm{~g}$, i). The subsequent mass spectrometry analysis revealed four main groups of potential interaction partners: cytoskeletal proteins (e.g., actin and tubulin), actin-binding factors (e.g., cofilin-1 and spectrin), AJ components (e.g., $\beta$-cat and p120) and factors involved in metabolism (e.g., pyruvate kinase and dihydropyrimidinase) (Fig. 3j, Supplementary Dataset 2). Among these factors, we found proteins that have been previously shown to directly interact with Limal, such as $\operatorname{actin}^{37}$, or participate together in a larger molecular complex, such as $\beta$-cat and p120 catenin ${ }^{7}$. Thus, altogether, the RNA-seq and proteomics analysis suggest that Limal might play a role in cytoskeletal dynamics as well as in cellular metabolism.

To examine the potential role of Limal in cell migration, we dissociated Lima1 KO and WT ESC and analysed their behaviour on a single-cell level. Strikingly, Limal KO ESC exhibited spherical membrane protrusions (blebs), that were rarely detectable in WT cells (Fig. 3k, Movies S1 and S2). The blebbing was exhibited only upon individualisation of the cells and it faded away completely, as the cells came together to form small colonies. Membrane blebbing has been subdivided into three phases, namely initiation (nucleation), expansion and retraction ${ }^{38}$, whereby bleb nucleation is established by cell membrane dissociation from the actin $\operatorname{cortex}^{39}$ or via local rupture of the cortex ${ }^{40,41}$. The expansion phase is driven by the hydrostatic pressure of the cytoplasm, followed by the formation of a new actin cortex under the blebbing membrane. Finally, the recruiting of myosin to the newly assembled cortex allows the bleb to retract $^{38}$.

To examine the blebbing in WT and Limal KO ESC, we stained the cells for the bleb marker phospho-ERM (ezrin, radixin and moesin $)^{38}$ and found that Limal KO ESC exhibit extensive pERMpositive membrane protrusions (Fig. 3L, white arrowheads). In nonblebbing WT ESC, Limal was uniformly localised on the cortex, which appeared as a continuous ring (Fig. $3 \mathrm{~m}$, blue arrowhead). However, in WT cells that formed blebs, Limal was absent from the blebbing membrane (Fig. 3m, yellow arrowheads). Accordingly, genetic deletion of Limal converted the overall non-blebbing ESC morphology to a highly blebbing phenotype (Fig. $3 \mathrm{k}, \mathrm{m}$ and $\mathrm{o}$ ). This indicates that Limal plays an integral role in stabilising the cortex, suppressing the formation of membrane blebs.

In contrast to the naïve ESC, primed pluripotent cells, such as conventional human ESC (hESC) and mouse epiblast stem cells (EpiSC), typically exhibit blebbing upon dissociation into single cells ${ }^{42}$. As bleb formation depends on actomyosin activation via the Rho/ROCK pathway ${ }^{38}$, treatment with a ROCK inhibitor 
a
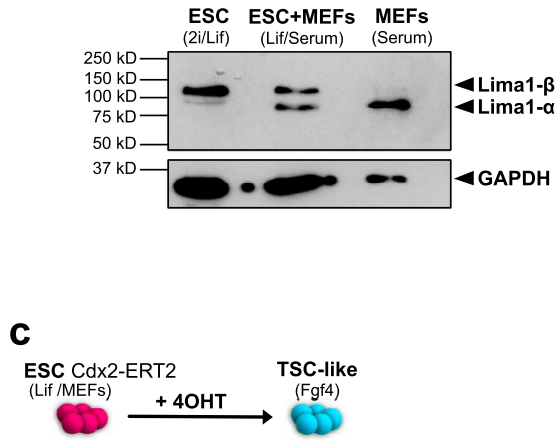

d

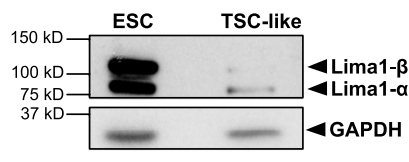

g

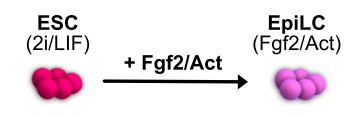

i

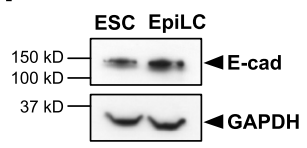

e

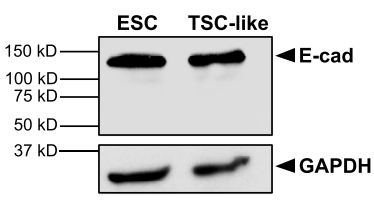

b
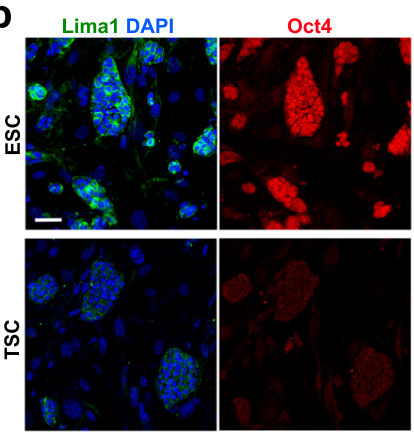

f
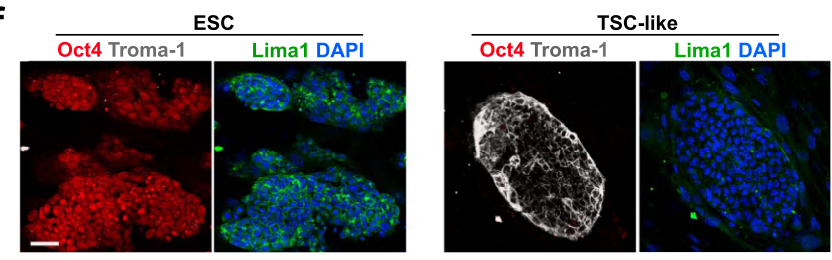

k

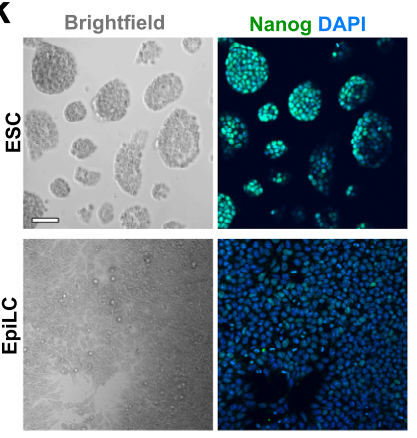

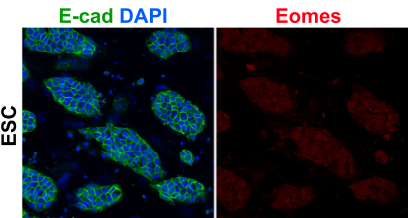

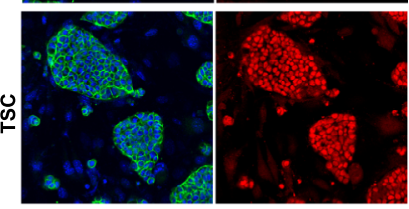

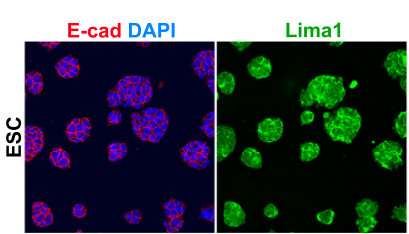

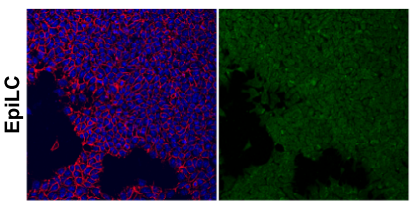

h

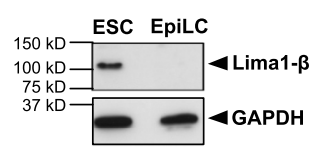

j

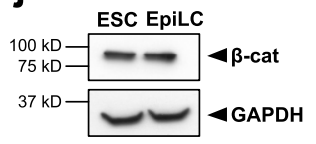

I

m

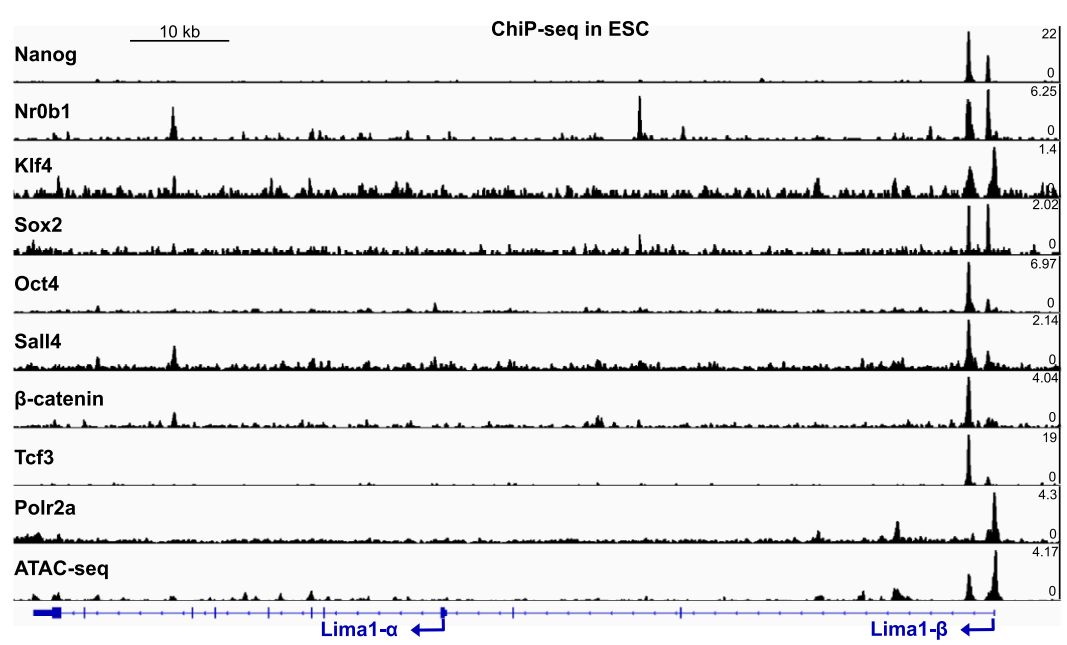

Fig. 2 Lima1 expression in embryonic and extraembryonic stem cell lines. a Western blot analysis of Lima1 expression in ESC and MEFs. b ESC and TSC stained for Lima1 and Oct4 (left panel) or E-cad and Eomes (right panel). c Schematic representation of ESC conversion to TSC-like cells. d Western blot analysis of Lima1 expression in ESC and TSC-like cells. e Western blot analysis of E-cad expression in ESC and TSC-like cells. $\mathbf{f}$ ESC and TSC-like cells stained for Oct4, Troma1 and Lima1. $\mathbf{g}$ Schematic representation of ESC conversion to EpiLC. $\mathbf{h}$ Western blot analysis of Lima1 expression in ESC and EpiLC. $\mathbf{i}$ Western blot analysis of E-cad expression in ESC and EpiLC. $\mathbf{j}$ Western blot analysis of $\beta$-cat expression in ESC and EpiLC. $\mathbf{k}$ ESC and EpiLC stained for Nanog (left panel) or E-cad and Lima1 (right panel). I Relative expression levels of Oct4, Nanog and Lima1 normalized to GAPDH in ESC and EpiLC. Mean values from three independent repetitions, data represent mean \pm SEM, unpaired Student's $t$-tests, 2-sided. $\mathbf{m}$ ChIP-seq peaks of pluripotency factors binding on the Lima1 locus in ESC. Polr2a peaks indicate the promoter region, ATAC-seq peaks indicate the accessible chromatin regions. Scale bars, (b), (f), (k), $50 \mu \mathrm{m}$. Experiments were repeated independently two times $(\mathbf{a}, \mathbf{b}, \mathbf{e}, \mathbf{f}, \mathbf{i}, \mathbf{j}, \mathbf{k})$ or three times (d, h) with similar results. Related to Figure $\mathrm{S} 2$. 
Lima1 locus

b

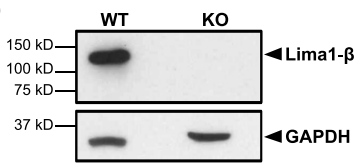

C
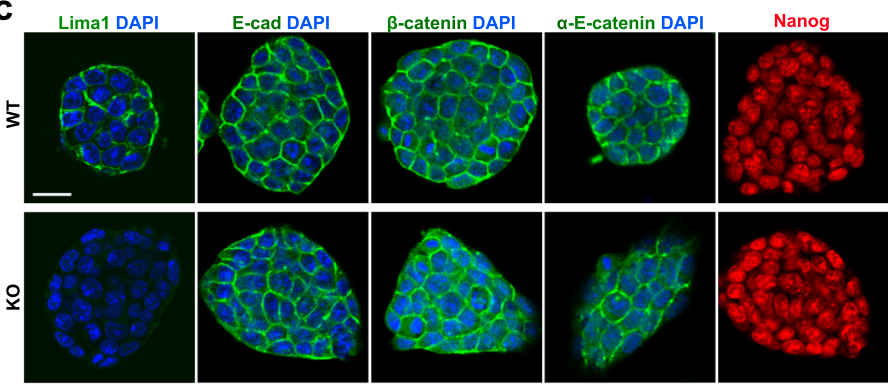

d

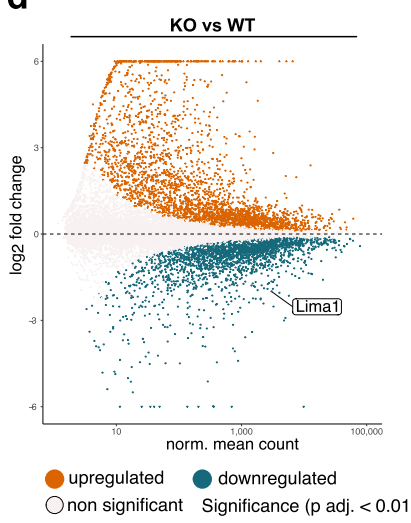

g

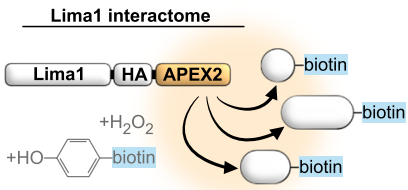

k

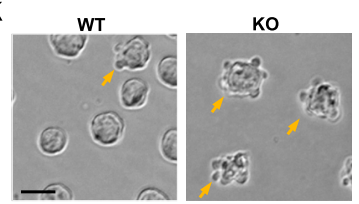

I

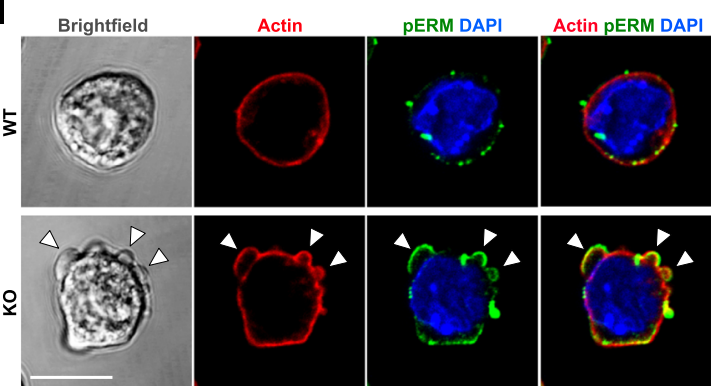

(Y-27632) has been shown to supress the blebbing of primed pluripotent cells ${ }^{42,43}$. Accordingly, Y-27632 treatment also diminished blebbing in Limal KO ESC (Fig. 3n, o). Altogether, this shows that Limal acts as an effector molecule downstream of the naïve pluripotency circuit, mediating the control of membrane dynamics in ESC.

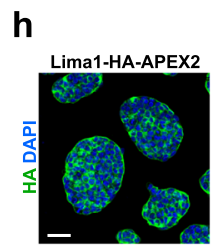

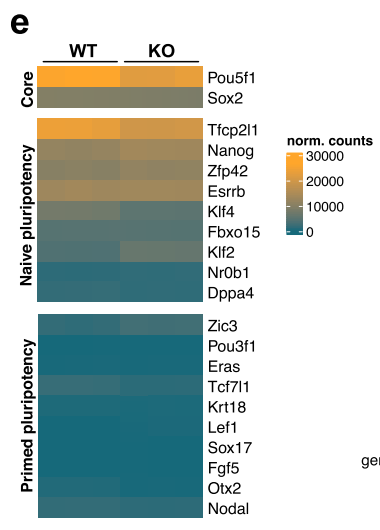
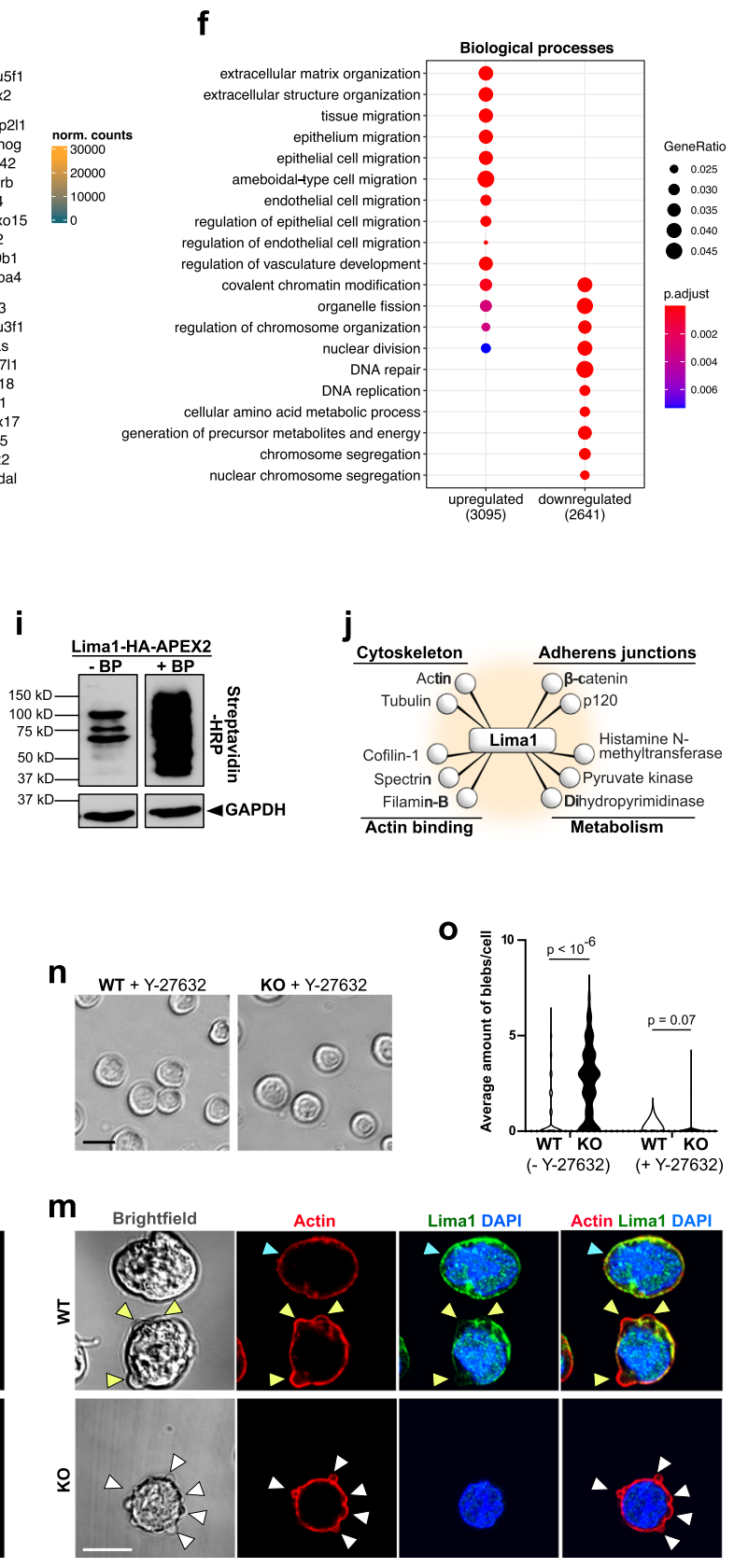

Limal depletion in ESC results in reduced mitochondrial ATP production, decreased rates of teratoma growth and a reduced contribution to chimeras. Pluripotent ESC can form noninvasive tumours (teratomas) that consist of derivatives of the three germ layers ${ }^{44}$. Loss of Limal has been implemented in malignancy ${ }^{10,14-17}$, but how Limal depletion affects the growth 
Fig. 3 Lima1 depletion in ESC. a CRISPR/Cas9 targeting strategy. b Validation of Lima1 deletion in ESC by western blot. c Lima1 KO and WT ESC stained for Lima1, E-cad, $\beta$-cat, $\alpha$-E-cat or Nanog. d MA plot of gene expression in Lima1 KO vs. WT ESC, cultured in N2B27 2i/Lif. Statistical testing for differential gene expression between conditions was performed using the default DESeq2 settings calculating the $p$-value using a two-tailed Wald-test with Benjamini-Hochberg correction for multiple testing and independent filtering of results. e Expression of core, naïve and primed pluripotency genes in WT and Lima1 KO ESC. f Gene ontology (GO) enrichment analysis "Biological processes". Gene Ratio indicates the fraction of genes comprised by the GO term, showing significant changes in their expression. $\mathbf{g}$ Scheme representation of APEX2 interactome analysis. h ESC expressing Lima1-HA-APEX2 construct, stained for HA. i Western blot analysis of Lima1-HA-APEX2 ESC cultured in the presence of or without biotin-phenol (BP). The biotinylated proteins are detected by streptavidin-HRP. $\mathbf{j}$ Example of putative Lima1 interaction partners identified by the APEX2 assay. $\mathbf{k}$ Membrane blebbing (arrows) in WT and Lima1 KO ESC. I WT and Lima1 KO ESC stained for actin, pERM and DAPI. Arrowheads indicate membrane blebs. $\mathbf{m}$ WT and Lima1 KO ESC stained for actin, Lima1 and DAPI. Blue arrowhead indicates Lima1 localisation on the cortex in control ESC; yellow arrowheads indicate loss of Lima1 on the membrane blebs in control ESC; white arrowheads indicate membrane blebs in Lima1 KO ESC. $\mathbf{n}$ WT and Lima1 KO ESC treated with Y-27632. $\mathbf{o}$ Mean distribution of cell membrane fluctuation counts per cell over $90 \mathrm{~s}$ of time-lapse imaging with $5 \mathrm{~s}$ intervals; $n=224$ (WT), 133 (WT treated), 260 (Lima1 KO), 159 (Lima1 $\mathrm{KO}$ treated) cells, Mann-Whitney tests, 2-sided. Scale bar, (c) $20 \mu \mathrm{m} ;(\mathbf{h}) 50 \mu \mathrm{m}$; (k), (I), (n), (o), $10 \mu \mathrm{m}$. Experiments were repeated independently two times $(\mathbf{c}, \mathbf{h}, \mathbf{l}, \mathbf{m})$ or at least three times $(\mathbf{b}, \mathbf{i}, \mathbf{k}, \mathbf{n}, \mathbf{0})$ with similar results. Related to Fig. S3.

of solid tumours is still obscure. To understand whether Lima1 depletion affects the growth rate and/or the composition of solid tumours, we subcutaneously injected WT ESC or Lima1 KO ESC into SCID mice to generate teratomas. Although Limal KO cells could give rise to teratomas, their size was substantially smaller compared to the tumours derived from WT ESC (Fig. 4a, b). Nevertheless, Lima1 KO teratomas consisted of tissue derivatives of all three germ layers, indicating that Limal depletion does not affect the ESC differentiation capacity (Fig. 4c).

To further explore the behaviour of tissues derived from Limal KO ESC, we generated chimeric embryos via aggregation of morulae with nuclear tdTomato-labelled Lima1 KO ESC or membrane tdTomato-labelled WT ESC. At E4.5, we found that similar numbers of Lima1 KO and WT cells were incorporated in the ICM of the chimeric blastocysts (Fig. 4d, e). However, after implantation, at E6.5 and later at E13.5, we found a gradual but substantial decrease of Limal KO cells (Fig. 4f-i). Despite the reduced number, the Limal $\mathrm{KO}$ cells were present in all examined foetal organs (Fig. 4j, k).

Next, we aimed to determine why the tumour growth and chimerism were reduced in Limal $\mathrm{KO}$ cells. To understand whether the initial pool of Lima1-deficient pluripotent cells may have survival and/or proliferative disadvantage, we compared the cell death and proliferation of Lima1 KO vs. WT ESC. We found that Limal depletion was not associated with an increase in apoptosis or necrosis (Fig. 5a). However, we found a decrease in the proliferation of Limal KO ESC cultured either in the presence of or without Y-27632 (Fig. 5b).

As the transcriptional profiling of Limal KO ESC and the APEX2 interactome analysis indicated an interplay of Lima1 and cellular metabolism, we asked whether the reduced cell proliferation of Lima1 KO ESC is caused by a metabolic defect. The APEX2 assay identified pyruvate kinase, which catalyses the last step of glycolysis, as a potential interaction partner of Limal. Nevertheless, we found that pyruvate kinase activity was not altered in Lima1 KO ESC (Fig. 5c).

Previous studies have shown that Limal accumulates in RasV12-transformed cells, which results in a decreased mitochondrial transmembrane potential $(\Delta \Psi \mathrm{m})$ in a non-cellautonomous manner ${ }^{45-47}$. Thus, we asked whether depletion of Limal affects the transmembrane potential and energy production of the mitochondria. We analysed the mitochondrial transmembrane potential using the tetramethyl-rhodamine ethyl ester (TMRE) assay and found that the TMRE signal was reduced in Limal KO ESC (Fig. 5d, e). As the energy required for ATP production is derived from the $\Delta \Psi \mathrm{m}^{48}$, this indicates that Limal KO ESC exhibit a decreased energy availability for ATP synthesis in a cell-autonomous fashion. The reduction in the $\Delta \Psi \mathrm{m}$ in Lima1 KO ESC did not result in an increase in the intracellular reactive oxygen species (Fig. 5f), suggesting that Limal depletion is not associated with mitochondrial oxidative damage.

Next, we conducted a mitochondrial stress test assay using an automatic flux analyser (Seahorse) to examine the oxygen consumption rate (OCR) in WT vs. Limal KO cells. This assay measures the OCR before and after applying inhibitors of different electron transport chain (ETC) components, thereby providing an assessment of multiple respiration parameters. We found that the basal and maximal respiration as well as the ATPlinked respiration and the non-mitochondrial oxygen consumption were substantially decreased in Limal KO cells (Fig. $5 g, \mathrm{~h}$ ). This shows that Lima1 depletion results in a general reduction of the mitochondrial ATP production rate and energy efficiency in a cell-autonomous manner. As it was previously reported that primed pluripotent cells exhibit low mitochondrial respiration ${ }^{49}$, we also compared the OCR of Lima1 KO ESC to WT EpiSC and found similar levels. Thus, although Lima1 KO ESC maintain the expression of naïve pluripotency transcription factors, they exhibit low mitochondrial respiration akin to the primed pluripotent state.

As the reduction in cell proliferation of Limal KO ESC was not compensated by the presence of Y-27632 (Fig. 5b), we examined whether the reduction of the OCR is also Y-27632-independent. Accordingly, we found similar OCR levels in Lima1 KO ESC cultured in the presence of or without Y-27632 (Fig. S4A). In addition, we differentiated WT and Lima KO ESC in vitro, using the embryoid body (EB) assay, and found no substantial difference in the OCR in WT and Limal KO EBs (Fig. S4B and $\mathrm{S} 4 \mathrm{C})$. This suggests that the Limal loss of function effects on the mitochondrial energetics are context-dependent and are associated with the undifferentiated ESC state.

Lima1-mediated suppression of membrane blebbing enables the incorporation of primed pluripotent cells into preimplantation embryos. Finally, we examined the gain of function effects of Limal expression in mouse and human primed pluripotent stem cells. To this end, we generated murine EpiSC and conventional hiPSC expressing the Limal-HA construct $(\beta$ isoform). These cells did not exhibit changes in the level of E-cad expression and adhesion, and they formed colonies that were morphologically undistinguishable from the control lines (Fig. 6a-d, S5A and S5B).

As the endogenous Limal is downregulated in primed cells, we asked whether the forced expression of Limal in this context results in the activation of naïve pluripotency makers. Therefore, we analysed the transcriptomes of control and Lima1-HA expressing EpiSC using RNA-seq (Fig. 6e-g, S5D-S5F, Supplementary Dataset 3). Principal component analysis revealed that the Lima1-HA EpiSC clustered together with the control EpiSC, 
a

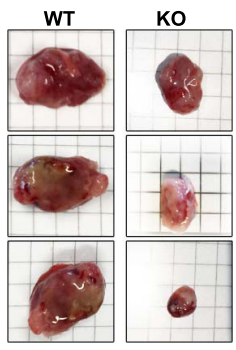

b

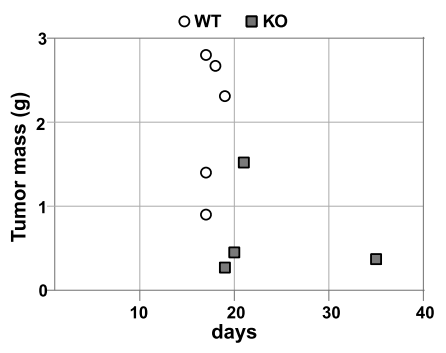

C

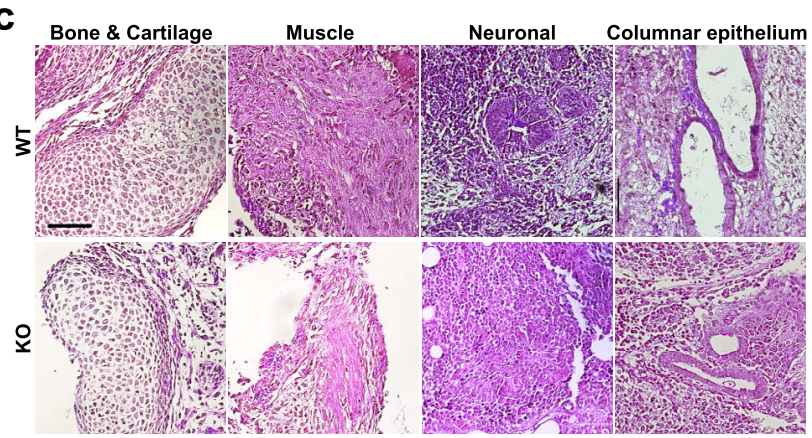

d

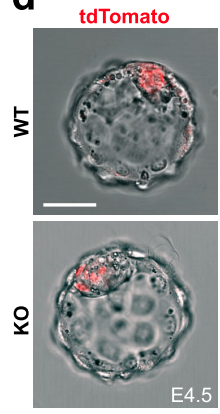

e

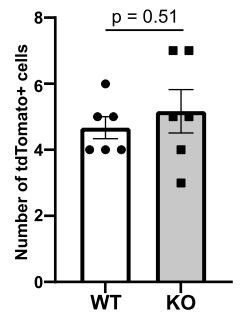

f

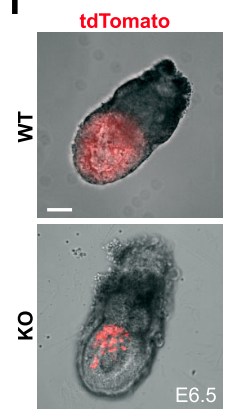

g

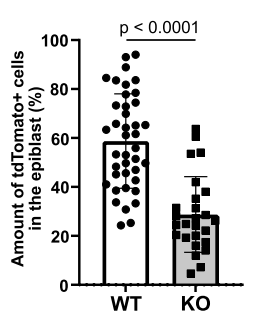

h

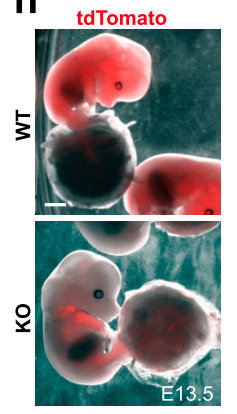

i

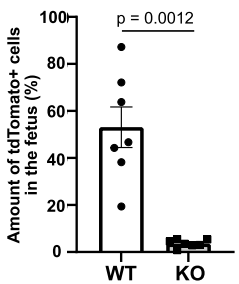

j

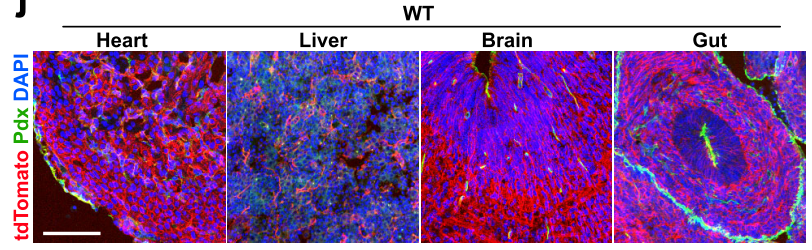

k

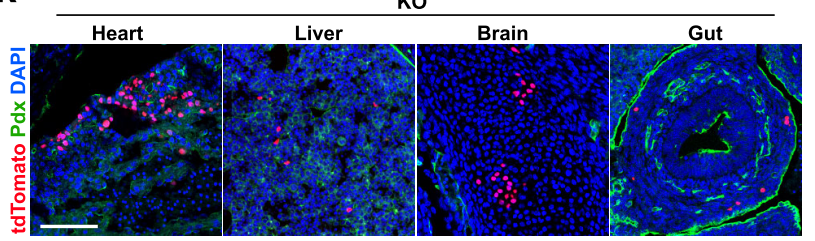

Fig. 4 Developmental potential of Lima1 KO ESC. a Teratomas derived from Lima1 KO or WT ESC. b Growth of WT and Lima1 KO teratomas.

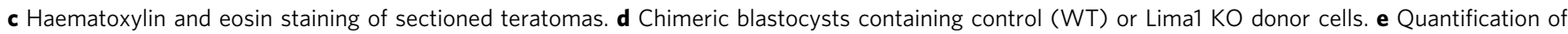
integrated donor cells at blastocyst stage, data represent mean \pm SEM, WT $n=6$ embryos, Lima1 KO $n=6$ embryos, unpaired Student's $t$-test, 2 -sided. f Chimeric E6.5 embryos containing control (WT) or Lima1 KO donor cells. $\mathbf{g}$ Quantification of the ectopic cells relative to the total epiblast cell number at E6.5. WT ( $n=40$ embryos), Lima1 KO ( $n=32$ embryos), three independent experiments. Data represent mean \pm SEM, Mann-Whitney test, 2 -sided. h Chimeric E13.5 embryos containing control (WT) or Lima1 KO donor cells. i Quantification of the ectopic cells relative to the total cell number of the foetus, determined by flow cytometry. WT ( $n=7$ embryos), Lima1 KO ( $n=7$ embryos), three independent repetitions. Data represent mean \pm SEM, unpaired Student's t-test, 2-sided. j Chimeric E13.5 embryos containing WT donor cells, stained for tdTomato, Podocalyxin and DAPI. k Chimeric E13.5 embryos containing Lima1 $\mathrm{KO}$ donor cells, stained for tdTomato, Podocalyxin and DAPI. Scale bars, (c) $50 \mu \mathrm{m}$; (d), $20 \mu \mathrm{m}$; (f) $50 \mu \mathrm{m}$; (h) $1000 \mu \mathrm{m}$; (j), (k), $100 \mu \mathrm{m}$; Experiments were repeated independently three times $(\mathbf{c}, \mathbf{j}, \mathbf{k})$ with similar results.

apart from the naïve ESC (Fig. 6e). Accordingly, gene expression analysis confirmed the ectopic upregulation of Limal (Fig. 6f), which was not associated with an upregulation of naïve pluripotency transcription factors or downregulation of primed pluripotency markers (Fig. 6g).

In addition, we characterised the transcriptional profiles of control (conventional) hiPSC and Limal-HA expressing hiPSC (Fig. 6h-j, S5G-S5I, Supplementary Dataset 4). We also included in the analysis available transcriptional data of naïve hiPSC ${ }^{50}$. The principal component analysis showed that Lima1-HA and control hiPSC clustered closely together, apart from the naïve hiPSC (Fig. 6h). Similar to the Lima1-HA EpiSC, the ectopic expression of Limal in conventional hiPSC was not associated with activation of naïve pluripotency genes or downregulation of primed pluripotency factors (Fig. 6i, j). Altogether, this shows that the expression of Limal-HA in both mouse and human primed pluripotent stem cells does not promote conversion to naïve pluripotency.
Next, we asked whether the ectopic expression of Limal in primed cells affects membrane blebbing. Control EpiSC and hiPSC formed blebs (Fig. 6k, o), which were efficiently suppressed by Y-27632 treatment (Fig. 61, p), in agreement with previous reports ${ }^{42,43}$. In contrast, blebbing was rarely observed in naïve hiPSC (Fig. 6n). We found that formation of blebs was substantially reduced as a result of the ectopic Limal expression in EpiSC and conventional hiPSC (Fig. 6k-m, o-q). In addition, studies have reported that the membrane blebbing in conventional human ESC (hESC) triggers an apoptotic response, whereas treatment with a ROCK inhibitor protects the dissociated hESC from cell death ${ }^{42,43}$. Accordingly, we found that suppressing membrane blebbing by Limal enhanced the survival of conventional hiPSC (Fig. S5C).

To further examine the effects of Limal expression on the membrane dynamics, we performed ultrastructural analysis to determine the subcellular localisation of Limal using immunogold labelling. We stained control and Limal-HA EpiSC for the 
a
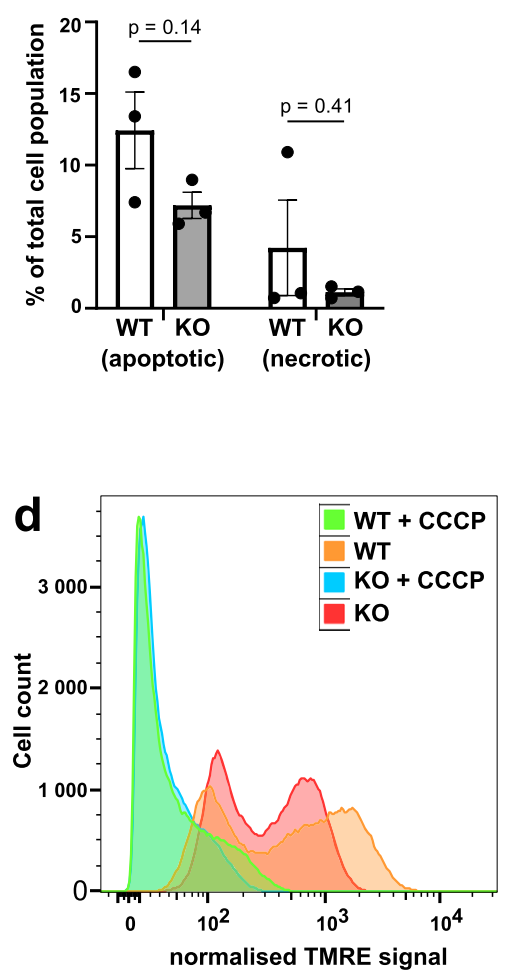

b

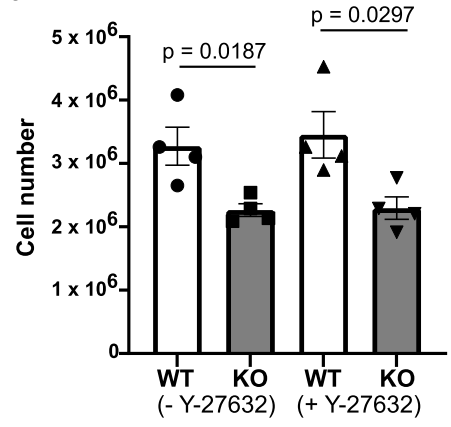

e

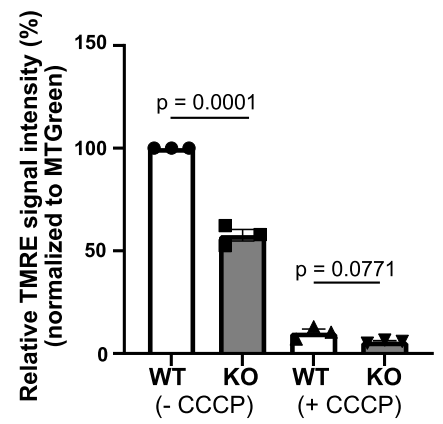

C

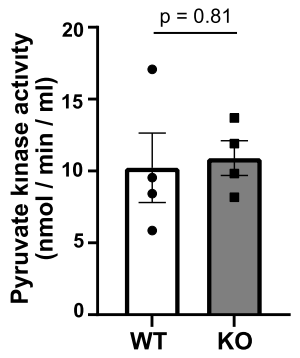

f

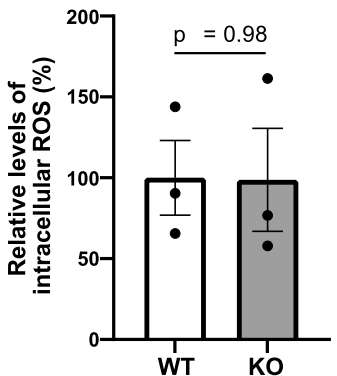

g

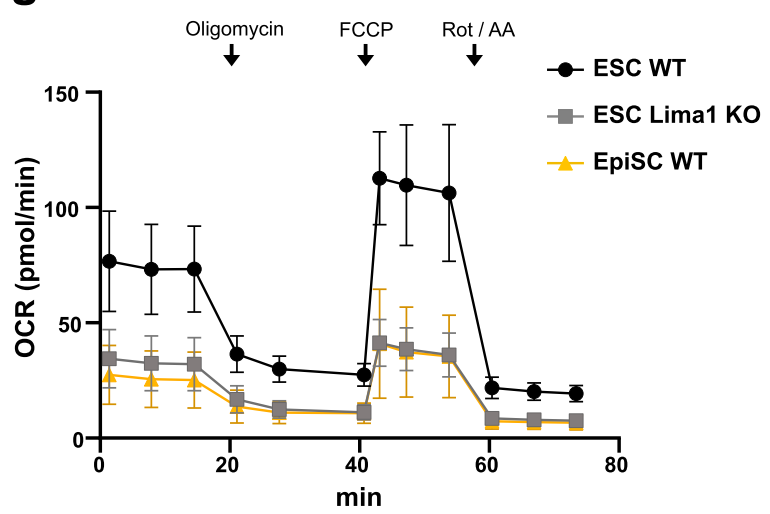

h

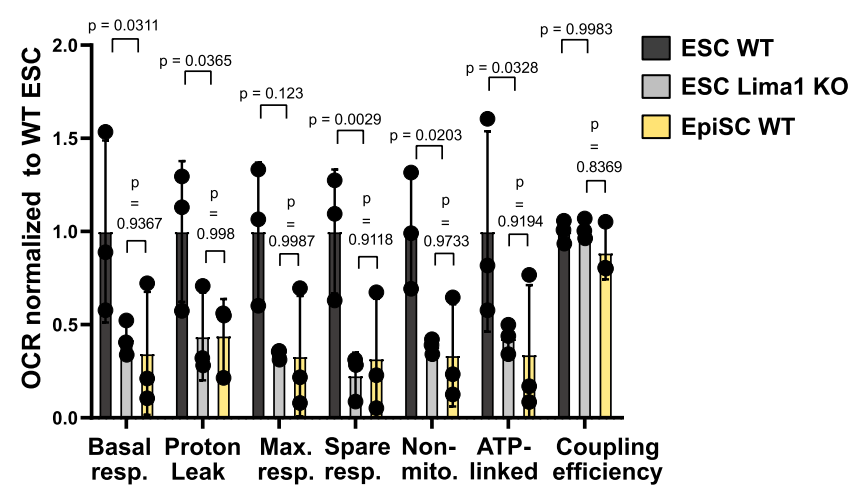

Fig. 5 Lima1 is required for proper mitochondrial energetics. a Proportion of apoptotic and necrotic Lima1 KO and WT ESC. The cells were individualized by trypsinization and cultured on cell-repellent plates for $2 \mathrm{~h}$ at $37^{\circ} \mathrm{C}$. After that, the cell death was determined by annexin $\mathrm{V}$ assay in combination with DAPI, three independent experiments, data represent mean \pm SEM, unpaired Student's $t$-test, 2-sided. b Quantification of WT and Lima1 KO cell proliferation after $96 \mathrm{~h}$ of culture. The cells were treated with or without Y-27632 upon dissociation. Untreated Lima1 KO ( $n=6$ samples) and WT ( $n=6$ samples), three independent experiments. Treated with Y-27632 Lima1 KO ( $n=6$ samples), WT ( $n=6$ samples), four independent experiments. Data represent mean \pm SEM, unpaired Student's $t$-tests, 2-sided. c Pyruvate kinase activity measured in WT and Lima1 KO ESC, $n=4$ independent experiments. Data represent mean \pm SEM, unpaired Student's $t$-test, 2-sided. d FACS analysis of WT and Lima1 KO ESC TMRE assay. Plot shows the TMRE signal normalized to the MitoTracker signal. CCCP is a mitochondrial oxidative phosphorylation uncoupler used as a negative control. e Quantification of TMRE signal intensity, normalized to MitoTracker signal intensity, $n=3$ independent experiments. Data represent mean \pm SEM, unpaired Student's $t$-test, 2-sided. f Quantification of relative intracellular ROS levels in Lima1 KO ESC, relative to WT ESC. Three independent experiments, data represent mean \pm SEM, unpaired Student's $t$-test, 2-sided. $\mathbf{g}$ OCR measurement using the Seahorse mitochondrial stress test assay. FCCP-Carbonyl cyanide-4 (trifluoromethoxy) phenylhydrazone; Rot Rotenone, AA-Antimycin. Three independent experiments, mean values \pm SEM. $\mathbf{h}$ Quantification of the OCR analysis, $n=3$ independent experiments. Data represent mean \pm SEM, 2-way ANOVA with Tukey's multiple comparisons. Related to Fig. S4. 
a

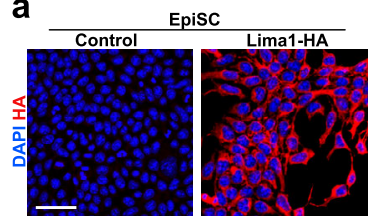

e

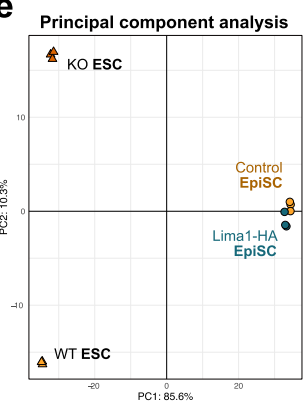

h

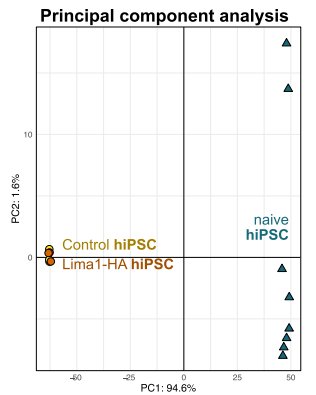

k

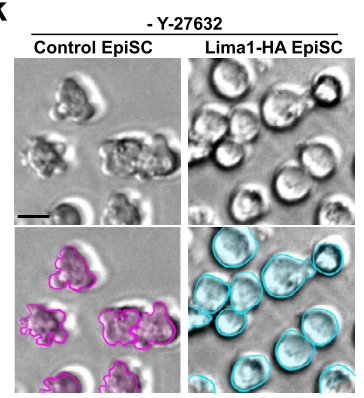

b

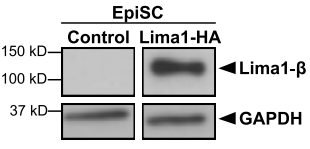

$f_{\text {Lima-HA EpiSC vs Control EpiSC }}$

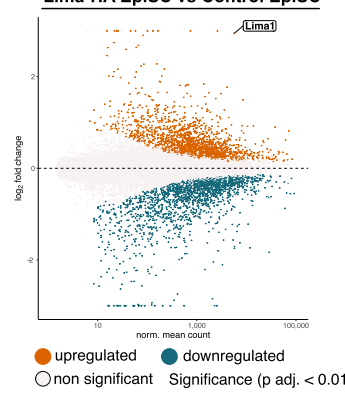

i

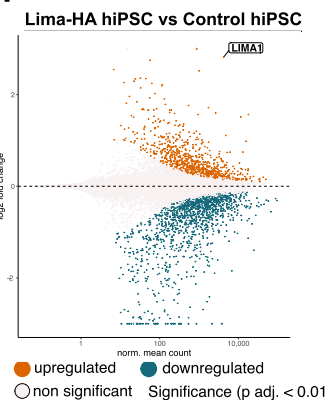

C

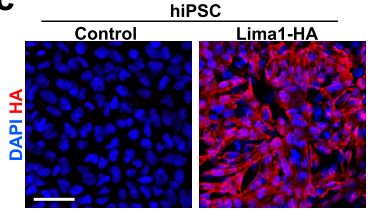

d

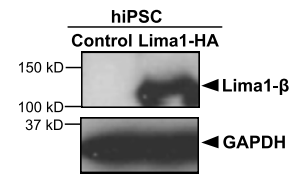

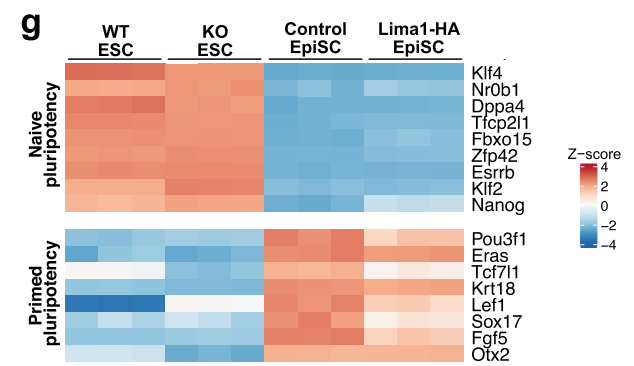

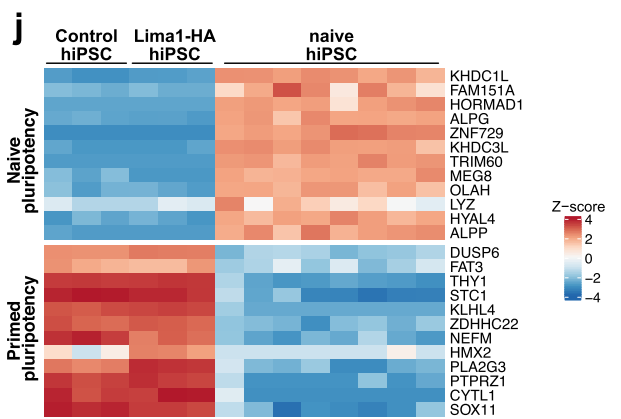

m

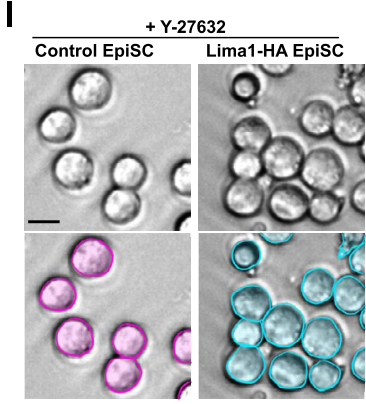

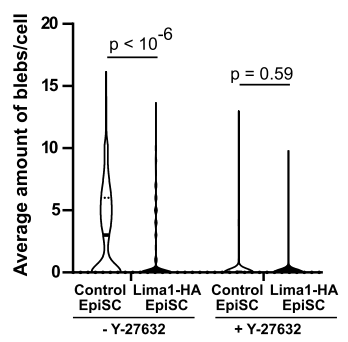

n

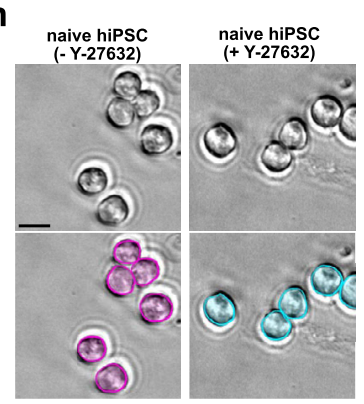

o

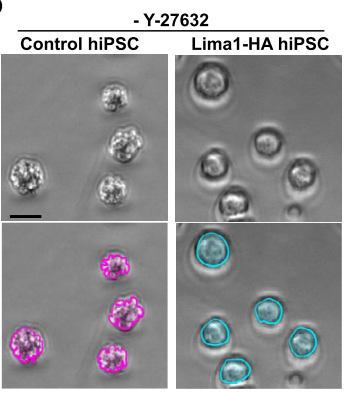

p

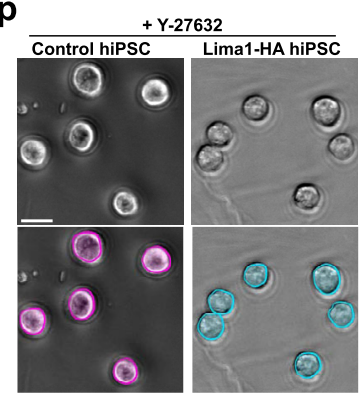

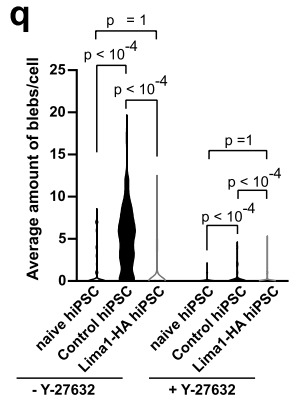

HA-tag and found Lima1-HA signal on the cell cortex (Fig. 7a). We did not detect enrichment of Limal in the mitochondria (Fig. 7a, b), suggesting that Limal is not directly involved in the mitochondrial composition. In addition, we examined the OCR in WT EpiSC or WT hiPSC vs. Lima-HA EpiSC or Lima-HA hiPSC, respectively and found no substantial difference between the control and Lima1-HA expressing cells (Fig. S6A-S6D), indicating that the effects of Limal on the mitochondrial energetics are associated with the naive pluripotent state.

To understand whether Limal is localised within the cortical actin, we carried out double immunogold staining using two different sizes of gold particles $-10 \mathrm{~nm}$ of diameter for detecting 
Fig. 6 Lima1 ectopic expression in primed pluripotent stem cells. a Control and Lima1-HA-expressing EpiLC stained for HA and DAPI. b Western blot analysis of Lima1 expression in control and Lima1-HA EpiSC. c Control and Lima1-HA-expressing conventional hiPSC stained for HA and DAPI. d Western blot analysis of Lima1 expression in control and Lima1 HA conventional hiPSC. e Principal component analysis of control EpiSC, Lima1-HA EpiSC, WT ESC and Lima1 KO ESC transcriptomes. $\mathbf{f ~ M A ~ p l o t ~ o f ~ g e n e ~ e x p r e s s i o n ~ i n ~ L i m a 1 - H A ~ v s . ~ c o n t r o l ~ E p i S C , ~ t w o - t a i l e d ~ W a l d - t e s t ~ w i t h ~ B e n j a m i n i - H o c h b e r g ~ c o r r e c t i o n ~}$ for multiple testing and independent filtering of results. $\mathbf{g}$ Gene expression of naïve and primed pluripotency markers in WT ESC, Lima1 KO ESC, control EpiSC, Lima1-HA EpiSC. h Principal component analysis of control conventional hiPSC, Lima1-HA hiPSC and naïve hiPSC (Giulitti et al., 2019)50

transcriptomes. i MA plot of gene expression in Lima1-HA vs. control conventional hiPSC, two-tailed Wald-test with Benjamini-Hochberg correction for multiple testing and independent filtering of results. $\mathbf{j}$ Gene expression of naïve and primed pluripotency markers control conventional hiPSC, Lima1-HA hiPSC and naïve hiPSC. $\mathbf{k}$ Dissociated control and Lima1-HA-expressing EpiSC. I Dissociated control and Lima1-HA-expressing EpiSC treated with Y-27632. m Mean distribution of cell membrane fluctuation counts per cell over $90 \mathrm{~s}$ of time-lapse imaging with $5 \mathrm{~s}$ intervals from $\mathbf{k}$ and $\mathbf{I} ; n>177$ cells for each condition, Mann-Whitney tests, 2-sided. $\mathbf{n}$ Dissociated naïve hiPSC cultured without or treated with Y-27632. o Dissociated control and Lima1-HAexpressing conventional hiPSC. p Dissociated control and Lima1-HA-expressing conventional hiPSC treated with Y-27632. q Mean distribution of cell membrane fluctuation counts per cell over $90 \mathrm{~s}$ of time-lapse imaging with $5 \mathrm{~s}$ intervals from $\mathbf{n}, \mathbf{0}$ and $\mathbf{p} ; n=92$ (Control hiPSC), 89 (Control hiPSC treated) 88 (naïve hiPSC), 100 (naïve hiPSC treated), 294 (Lima1-HA hiPSC), 245 (Lima1-HA hiPSC treated) cells; 2-way ANOVA with Tukey's multiple comparisons. Scale bars, (a), (c), $50 \mu \mathrm{m} ;(\mathbf{k}),(\mathbf{I}),(\mathbf{n}),(\mathbf{o}),(\mathbf{p}), 10 \mu \mathrm{m}$. Experiments were repeated independently two times (a, b, c, d) or three times (m, q) with similar results. Related to Fig. S5.

Limal and $15 \mathrm{~nm}$ of diameter for detecting actin. The transmission electron microscopy confirmed that Limal was enriched on the cortical actin filaments (Fig. 7b), in accord with the proposed role of Limal in stabilisation of the cortex, supressing the formation of membrane blebs.

Limal protein harbours two actin-binding domains localised in the $\mathrm{N}$-terminal and C-terminal regions, as well as a central LIM domain, which was suggested to mediate Limal dimerization ${ }^{8-10}$. To examine the role of these domains on the membrane dynamics, we generated deletion constructs, lacking the $\mathrm{N}$-terminal or the C-terminal regions, or the LIM domain and expressed these constructs in EpiSC (Fig. 7c, d). In comparison to the full-length Lima1-HA, $\mathrm{N}$ - and C-terminal deletions, as well as monomeric Lima1 ( $\triangle \mathrm{LIM})$, failed to suppress membrane blebbing (Fig. 7e). Likewise, tethering Limal to the cell membrane by fusing the full-length Limal to E-cad (lacking the intracellular domain) was also inefficient to abolish blebbing (Fig. 7c-e). This indicates that the cytoplasmic pool of dimeric Limal proteins, bound to the cortical actin filaments, suppresses the formation of membrane blebs in pluripotent stem cells.

The establishment of a primed pluripotent state also results in a reduced incorporation efficiency of EpiSC and hiPSC into preimplantation embryos. To understand whether suppressing the membrane blebs via forced expression of Limal allowed for EpiSC integration into early embryos, we aggregated control or Lima1-HA EpiSC with 8-cell stage morulae. We found a substantial increase in the number of chimeric blastocysts when we used donor EpiSC expressing Limal-HA for aggregation (Fig. $8 \mathrm{a}-\mathrm{c}$ ). Moreover, ectopic upregulation of Limal in conventional hiPSC enabled the generation of chimeric blastocysts (Fig. 8d-f). Alternatively, supplementing the embryo culture medium used for morula aggregation with a ROCK inhibitor allowed the control EpiSC or hiPSC to be incorporated into preimplantation embryos (Fig. S7A-S7D). Altogether, this shows that suppression of membrane blebbing by Limal is sufficient to enable primed pluripotent cells engraftment into murine preimplantation embryos.

\section{Discussion}

Lima1 is an epithelial actin-binding protein that has been implicated and extensively studied in the progression of various types of cancers ${ }^{8}$. It also binds $\alpha$-cat and associates with the AJ complex on the cell membrane ${ }^{7,13}$. We found that in the context of the developing mouse embryo, Limal exhibits a pattern of expression that is not confined only to epithelial compartments.
A typical feature of the AJ complex proteins E-cad and N-cad is their almost mutually exclusive expression pattern. Similarly, $a$ E-cat and $\alpha-\mathrm{N}$-cat are preferentially found in complex with E-cad and $\mathrm{N}$-cad, respectively, whereas $\beta$-cat exhibits very broad expression, as it binds directly to both cadherins and also plays a central role in the Wnt signalling pathway. During EMT, E-cad is downregulated and $\mathrm{N}$-cad upregulated in a process known as cadherin switch ${ }^{1}$. Unexpectedly, we found Limal localisation in both E-cad and N-cad expression domains, overall resembling the $\beta$-cat expression pattern. However, our immunohistochemistry analysis does not differentiate between the long or short isoforms of Lima1. Therefore, Lima1- $\alpha$ and Lima1- $\beta$ may still exhibit an unaccounted differential expression pattern in the foetus. Moreover, we found $\beta$-cat and Tcf3 binding on the Lima1- $\beta$ promoter region, which indicates that in certain contexts, Lima1- $\beta$ could be a direct target of the Wnt pathway. As Wnt signalling is aberrantly activated in cancer, this may help explain why upregulation of Lima1- $\beta$ is observed in some breast cancer cells lines, where the shorter isoform is depleted ${ }^{10}$.

We found that the Limal transcripts and protein are already present in the oocyte before fertilisation. During the transition from the GV to MII stage, Limal accumulates on the membrane above the spindle and later in the polar body. This specialised membrane domain, known as cortical actin cup, plays a critical role in the asymmetric division during meiosis, which results in the extrusion of the polar body ${ }^{51}$. Several actin nucleators, such as ARP2/3 complex and formin-2, regulate the cytoskeletal remodelling during oocyte maturation ${ }^{52,53}$, and it would be interesting to address the potential role of Limal in this process, which would require depleting the maternal transcripts. Interestingly, Limal has already been shown to localise in the cleavage furrow in HeLa cells, where it is involved in the maintenance of the contractile ring ${ }^{54}$. Therefore, Limal might also play a role during meiosis, particularly in the process of the polar body extrusion.

At the 16-cell stage, we found that Limal expression is enriched in the inside cells of the morula. These cells give rise to the ICM of the blastocyst, whereas the outside cells form the $\mathrm{TE}^{55}$. In contrast, E-cad, $\beta$-cat and $\alpha$-E-cat exhibited overall uniform expression in the early lineages. Accordingly, Limal was also highly expressed in ESC, whereas E-cad and $\beta$-cat showed similar levels of expression in both ESC and TSC. Moreover, E-cad and $\beta$-cat expressions were not affected by the exit of naive pluripotency and the establishment of a primed state, whereas Limal was downregulated in response of this transition. Essentially, we found that the Limal promoter region is bound by several pluripotency transcription factors, such as Nanog, Nr0b1, Klf4, Sox2, Oct4, Sall4, as well as $\beta$-cat and Tcf3, indicating 
a
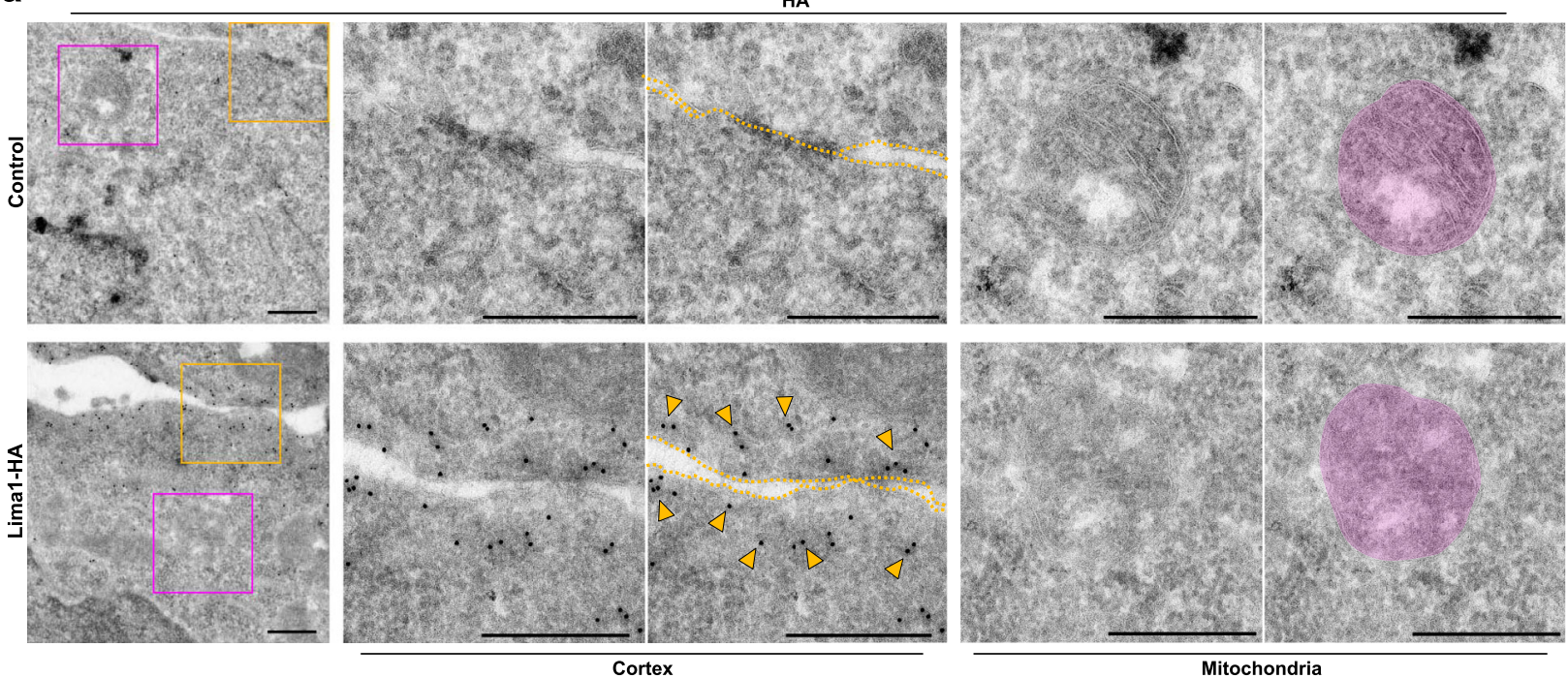

b

Actin $(15 \mathrm{~nm} \varnothing) \operatorname{Lima} 1(10 \mathrm{~nm} \varnothing)$
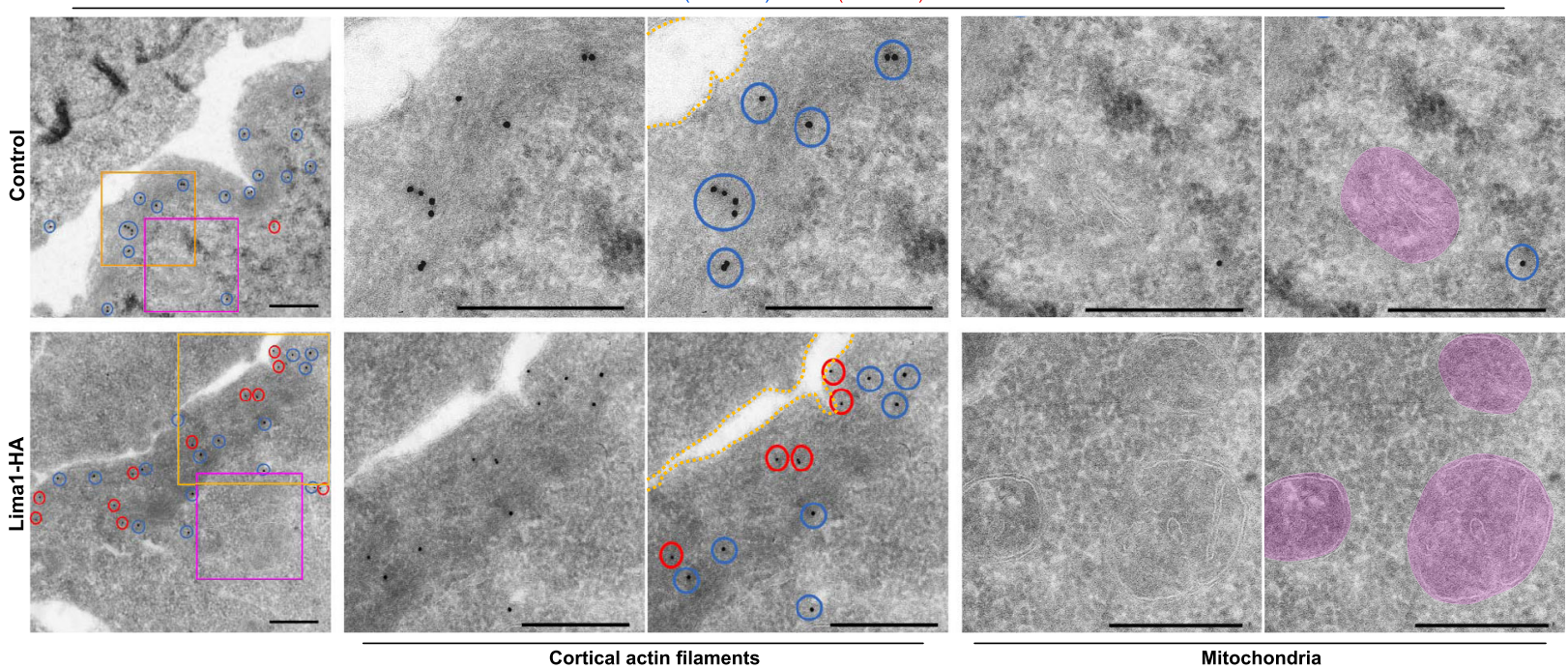

C

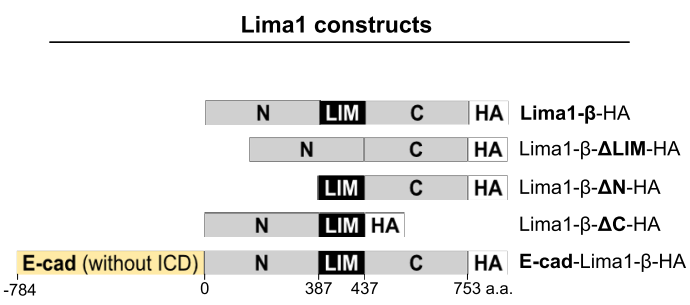

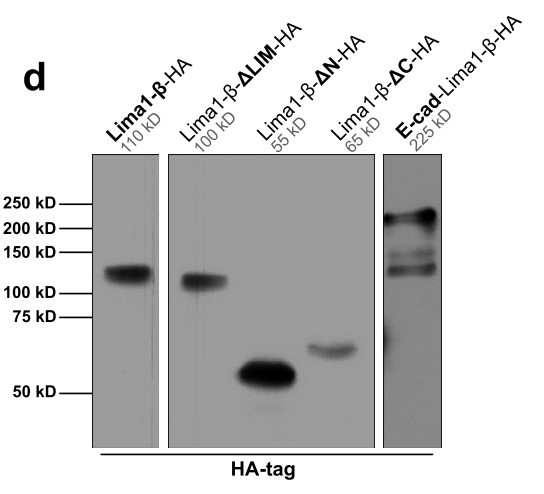

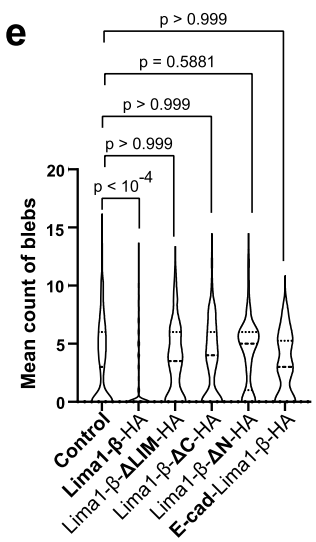

that Limal expression is under the control of the naïve pluripotency network (Fig. 9). This suggests that the establishment of the pluripotent fate activates Limal expression in the inner cells of the early embryo.

At mid and late blastocysts stage, we found that the epiblast cells retain a relatively high level of Lima1, whereas the PE cells appeared to decrease Limal expression. A recent study of Yanagida et al. showed that the PE cells exhibit larger surface fluctuations (membrane blebs), compared to the epiblast cells. Based on these results, a model was proposed suggesting that the difference in the membrane dynamics is involved in the segregation of PE and epiblast cells during blastocyst maturation ${ }^{56}$. 
Fig. 7 Ultrastructural analysis and expression of Lima1 deletion constructs in EpiSC. a Transmission electron microscopy of immunogold staining for the HA-tag in control and Lima-HA EpiSC. Arrowheads indicate the HA signal; mitochondria are marked in magenta. At least three technical replicates with similar results. b Transmission electron microscopy of double immunogold staining for Lima1 and Actin in control and Lima-HA EpiSC. Blue and red circles indicate the $15 \mathrm{~nm}$ and $10 \mathrm{~nm}$ gold particles, respectively; mitochondria are marked in magenta. At least three technical replicates with similar results. c Maps of the Lima1 constructs. $\mathbf{d}$ Western blot analysis of the expression of the Lima1 constructs detected by anti-HA antibody. e Mean distribution of cell membrane fluctuation counts per cell over $90 \mathrm{~s}$ of time-lapse imaging with $5 \mathrm{~s}$ intervals; $n=225$ (Control), 245 (full-length Lima1- $\beta$ ), 68 (del LIM domain), 78 (del C-terminus), 51 (del N-terminus), 18 (E-cad-Lima1- $\beta$ fusion) cells, one-way ANOVA. Scale bars, (a), (b) $1 \mu \mathrm{m}$. Related to Fig. S6.

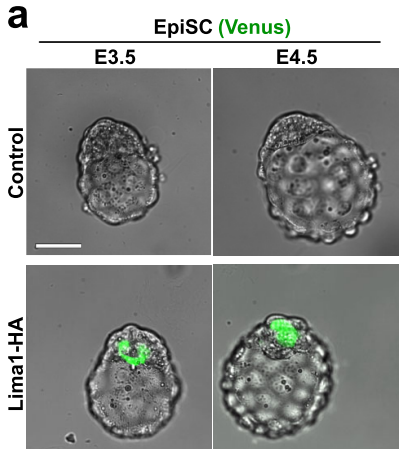

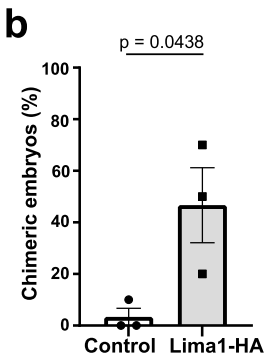

C

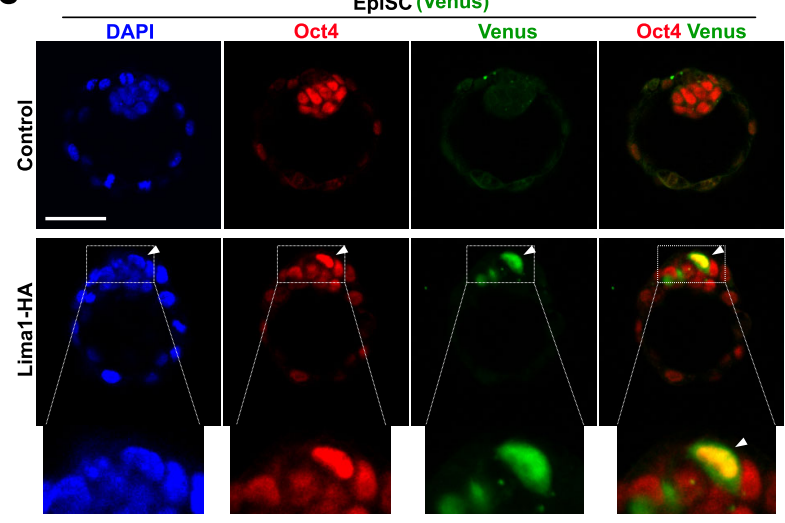

e

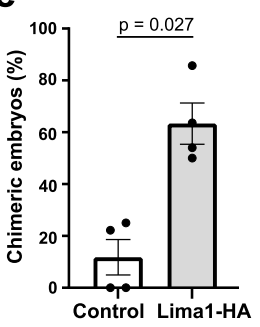

f

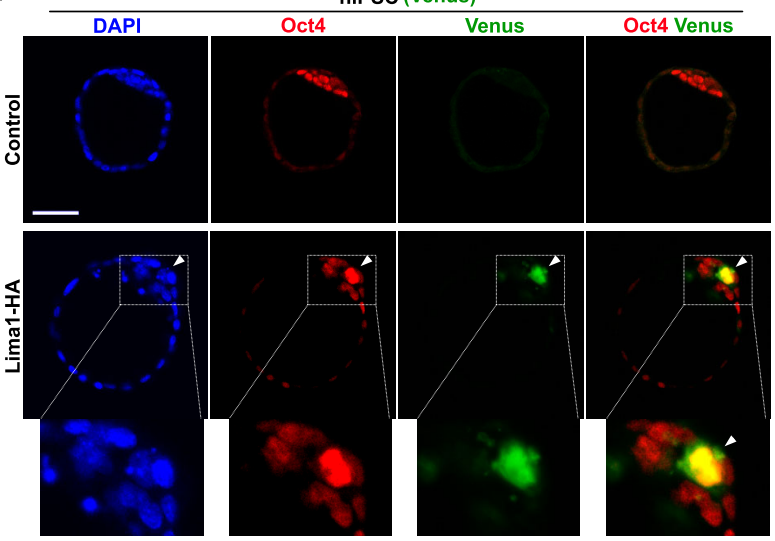

Fig. 8 Engraftment of Lima1-HA-expressing primed pluripotent cell in mouse pre-implantation embryos. a E3.5 and E4.5 chimeric blastocysts generated via morula aggregation using control or Lima1-HA-expressing EpiSC. b Quantification of the chimeric blastocysts containing control (22 embryos) or Lima1HA-expressing (26 embryos) EpiSC at E4.5. Data represent mean \pm SEM, $n=3$ independent experiments, unpaired Student's $t$-test, 2 -sided. c Chimeric blastocysts generated via morula aggregation using control (upper panel) or Lima1-HA-expressing EpiSC (lower panel) and stained for Venus, Oct4 and DAPI. Arrowhead indicates integrated EpiSC. d E3.5 and E4.5 chimeric blastocysts generated via morula aggregation using control or Lima1-HA-expressing conventional hiPSC. e Quantification of the chimeric blastocysts containing control (30 embryos) or Lima1-HA-expressing (33 embryos) conventional hiPSC at E4.5. Data represent mean \pm SEM, $n=4$ independent experiments, Mann-Whitney test, 2-sided. f Chimeric blastocysts generated via morula aggregation using control (upper panel) or Lima1-HA-expressing conventional hiPSC (lower panel) and stained for Venus, Oct4 and DAPI. Arrowhead indicates integrated hiPSC. Scale bars, (c), (f), $20 \mu \mathrm{m}$. Experiments were repeated independently three times (a, c, d) with similar results. Related to Fig. S7.

The PE cells displayed also elevated levels of pERM ${ }^{56}$, similarly to our observations of pERM enrichment in the ameboid membrane protrusions in Lima1 KO ESC. Thus, a reduction of Lima1 expression may serve as an indicator of cells that exhibit dynamic membrane fluctuations. Moreover, the progressive changes in the expression pattern of Limal in the PE and epiblast cells of the ICM may contribute to a surface fluctuations-driven segregation of these lineages, which as proposed by Yanagida et al., resembles phase separation between active and passive particles in colloidal mixtures ${ }^{56}$.

Loss of Limal did not affect the expression of pluripotency markers nor the differentiation capacity of the ESC. However, the growth of the Lima1 $\mathrm{KO}$ teratomas, as well as the proportion of donor Lima1 $\mathrm{KO}$ cells in post-implantation embryos, were severely reduced. ESC exhibit bivalent metabolism, using both OxPhos and glycolysis, whereas somatic cells rely mainly on OxPhos for ATP production to support their growth ${ }^{57}$. In addition, the transition from a naïve to a primed state of pluripotency results in the reduction of OxPhos and a switch mainly to glycolysis $49,58,59$. Loss of Lima1 did not cause cells to enter into primed pluripotency, but the mitochondrial ATP production rate was substantially reduced. Accordingly, Lima1 KO ESC exhibited decreased proliferation rate, which may contribute to the overall smaller size of the teratomas. The exact underlying molecular 

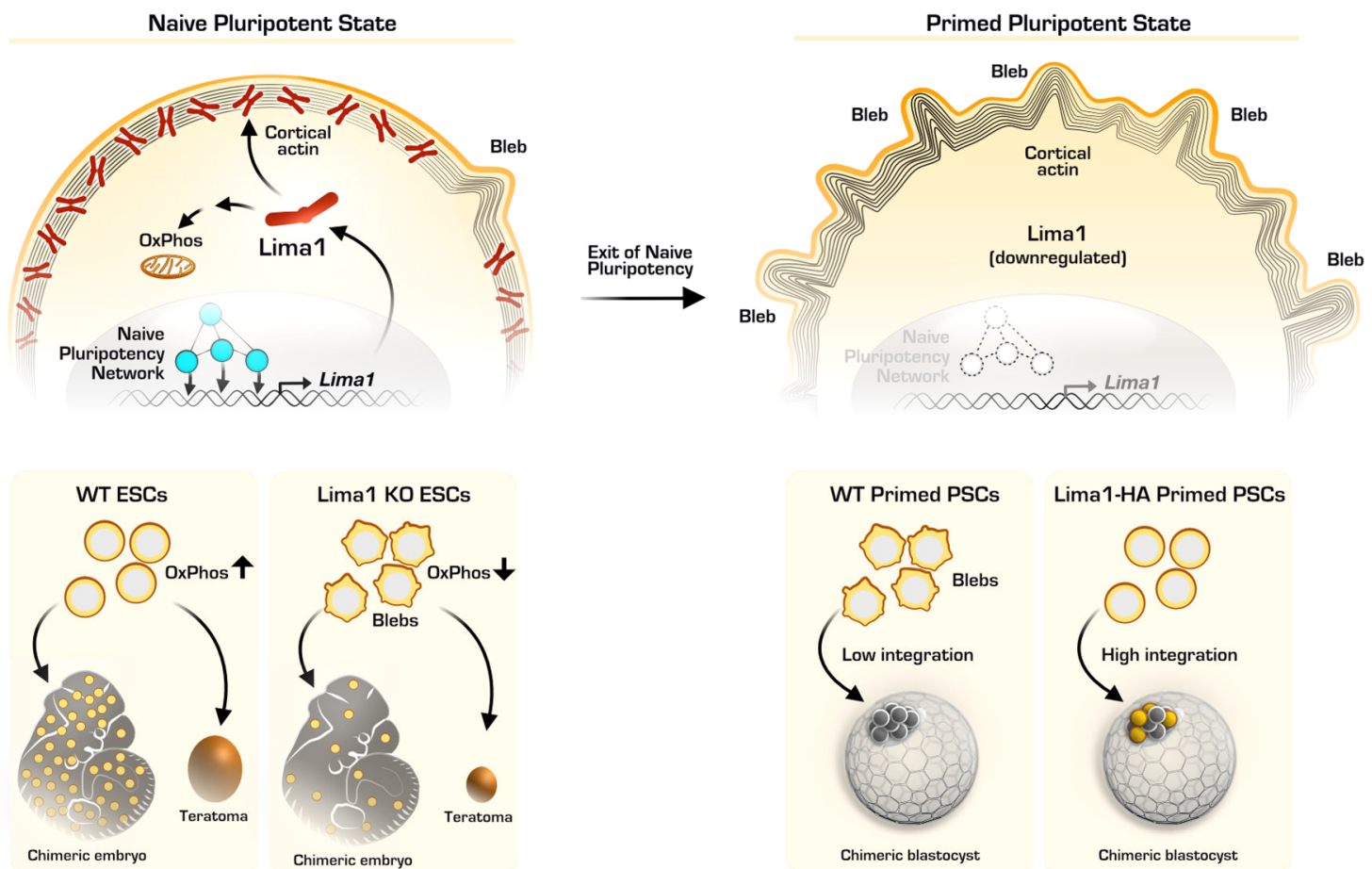

Fig. 9 Lima1 mediates the pluripotency control of membrane dynamics and cellular metabolism. Lima1 expression is promoted by the naïve pluripotency transcription factors, which occupy the Lima1 promoter region. In turn, the cytoplasmic pool of dimeric Lima1 proteins bound to the actin filaments stabilises the cortex, thereby suppressing the formation of membrane blebs in naïve pluripotent cells. Lima1 is also involved in the mitochondrial energetics and is crucial for growth of teratomas and embryonic chimerism of ESC. Upon exit of naïve pluripotency, the naïve transcriptional circuit is dismantled and Lima1 is downregulated, resulting in the formation of membrane blebs. Accordingly, ectopic expression of Lima1 in mouse and human primed pluripotent cells suppresses membrane blebbing and enables the incorporation of the primed cells into murine pre-implantation embryos. OxPhos, oxidative phosphorylation; naïve pluripotency transcription factors are marked in blue; donor cells are marked in yellow.

mechanism remains open. As Limal exhibited a broad expression in the foetus, loss of Limal may affect actin dynamics, membrane properties and adherens junctions in various tissues, which may also account for the reduced tumour growth and chimerism. In addition, Limal KO cells might face cell competition and potentially be outcompeted by the host embryo's cells, as a recent study showed that cells with mitochondrial dysfunction are eliminated in the post-implantation conceptus ${ }^{60}$.

Previously, Zhang et al. reported a conditional deletion of exon 5 of the Limal locus via adenoviral EIIa promoter-directed Cre recombinase ${ }^{61}$ for the generation of heterozygous mice, which exhibited lower cholesterol absorption and decreased plasma total cholesterol levels ${ }^{19}$. The homozygous exon $5 \mathrm{del} / \mathrm{del}$ mice were viable and also displayed decrease cholesterol absorption ${ }^{19}$. Here, in addition to exon 5 , we deleted also exon 4 , which contains the second transcription start site of the Limal locus. Whether this targeting strategy may result in an embryonic phenotype remains to be determined. Alternatively, Lima1 KO cells may exhibit a developmental disadvantage in vivo only in a competitive environment, such as in the context of chimeric embryos.

Understanding how Lima1 regulates mitochondrial function on a molecular level will require further investigation. Although we found several putative interaction partners involved in cellular metabolisms, such as pyruvate kinase and dihydropyrimidinase, none of these candidates directly control any key steps of energy homeostasis. Thus, it is possible that a yet unidentified Limal interaction partner(s) is involved in this process. Alternatively, the primary function of Limal as an actin-binding protein may indirectly affect the cellular metabolism. For instance, it has been shown that actin cytoskeleton dynamics regulate the activity of aldolase A, which is a key glycolytic enzyme ${ }^{62}$. In addition, application of force to the E-cad complex promotes the activity of AMP-activated protein kinase (AMPK), which plays a critical role in glucose and fatty acid uptake and oxidation ${ }^{63}$. As Limal can act as a mechanosensor ${ }^{13}$, potential changes in the mechanotransduction in Lima1 KO cells may also, in turn, influence the cellular metabolism.

Lima1 KO ESC exhibited membrane blebbing similarly to primed pluripotent cells, where the endogenous Lima1 expression was downregulated upon exiting the naïve state (Fig. 9). Ectopic expression of Limal in EpiSC and conventional hiPSC was sufficient to stabilise the actin cortex and prevent blebbing nucleation. A stabilising role of Limal on the cortex has also been reported in human umbilical vein endothelial cells (HUVEC), where downregulation of Limal results in gaps on the cortical actin $^{64}$. Interestingly, HUVEC form classical lamellipodia and junction-associated intermittent lamellipodia in which Lima1- $\alpha$ controls the protrusion dynamics, whereas Lima1- $\beta$ binds and stabilizes stress fibres ${ }^{37}$. Moreover, it was reported that Erkmediated phosphorylation of Limal reduces the affinity for actin filaments, which contributes to cell motility ${ }^{65}$. In $2 \mathrm{i} / \mathrm{Lif}$ culture conditions, Erk activity is suppressed by the chemical inhibition of the upstream Mek, whereas in primed pluripotency culture conditions, Erk activity is promoted by the stimulation of the Fof signalling 22,26 . Thus, although, Limal is transcriptionally downregulated upon exiting naïve pluripotency, we cannot exclude that additional fine-tunning of Limal-actin interactions occur on a post-translational level by the Fgf/Erk pathway.

We found that the Lima1-mediated suppression of membrane blebbing enabled primed pluripotent cells to be integrated into murine pre-implantation embryos. Interestingly, E-cad overexpression has also been shown to promote EpiSC 
incorporation ${ }^{66}$, but we found no substantial changes in the endogenous E-cad level upon exit of naïve pluripotency or upon ectopic expression of Lima1 in EpiSC. Nevertheless, the stabilisation of the actin cortex by Limal gain of function may promote more stable F-actin engagement with the E-cad complex, thus resembling the net effect of E-cad overexpression. In addition, it has been shown that human pluripotent stem cells can be integrated into pre-implantation mouse embryos via suppression of cell death ${ }^{67-69}$. Accordingly, Limal expression, as well as treatment with a ROCK inhibitor, promoted the survival of dissociated hiPSC. This indicates that downregulation of the endogenous Limal in primed hiPSC, which results in membrane blebbing and apoptotic response, is a key part of the interspecies engraftment barrier.

In tumour cells, membrane blebbing can mediate cell motility, which is used by the malignant cells as an alternative mechanism of migration ${ }^{41,70}$. This alteration in the morphology and behaviour of the cancer cells is known as a mesenchymal-amoeboid transition $(\mathrm{MAT})^{70}$. As blebbing motility does not require degradation of the extracellular matrix, MAT allows tumour cells to evade anti-cancer treatments, which rely on pharmacological protease inhibitors ${ }^{70,71}$. Our results in pluripotent cells show that Limal depletion enables blebbing and suggest that in pathological conditions, this may promote tumour resistance to protease inhibitors. Thus, treating malignancies associated with Limal deficiency may benefit from cancer therapeutics that target regulators of membrane blebbing.

\section{Methods}

Mice. Animal experiments and husbandry were performed according to the German Animal Welfare guidelines and approved by the Landesamt für Natur, Umwelt und Verbraucherschutz Nordrhein-Westfalen (State Agency for Nature, Environment and Consumer Protection of North Rhine-Westphalia), protocol number 84-02.04.2016.A186. The mice used in the study were at ages ranging from 6 weeks to 5 months. The animals were maintained at $21.5^{\circ} \mathrm{C}, 55-60 \%$ humidity under a 14-h light/10-h dark cycle with free access to food and water. Male mice were kept individually, whereas the female mice were housed in groups of up to four per cage. Embryos for experiments were obtained from wild-type CD1 and B6C3F1 from matings using females with natural ovulation cycles or after superovulation.

Cell culture. DR-4 or CF-1 MEFs (gift from Prof. Dr. Hans R. Schöler) were cultured on gelatin-coated plates in DMEM medium (Sigma, D5671), supplemented with 15\% FCS (Biochrome, S0615), $10 \mathrm{U} / \mathrm{ml}$ penicillin $/ 50 \mu \mathrm{g} / \mathrm{ml}$ streptomycin solution (Sigma, P4333), 2 mM L-glutamine (Sigma, G7513), $1 \mathrm{mM}$ sodium pyruvate (Sigma, S8636), 1x MEM non-essential amino acids (Sigma, M7145) and $0.15 \mathrm{mM} \beta$-mercaptoethanol (Sigma, M3148). MEFs were mitotically inactivated by exposure to $10 \mu \mathrm{g} / \mathrm{ml}$ mitomycin C (Sigma, M0503) for $2 \mathrm{~h}$, before splitting for coculture experiments.

Mouse ESC-E14 (gift from Prof. Dr. Hans R. Schöler), R1 (gift from Prof. Dr. Rolf Kemler) or $\mathrm{mT} / \mathrm{mG}^{72}$, were grown on DR-4 feeders in MEF medium supplemented with $1000 \mathrm{U} / \mathrm{ml}$ Lif (home-made). The cells were split every 2-3 days using $0.25 \%$ Trypsin-EDTA (Gibco, 25200056). For experiments requiring ground state culture conditions, the ESC were cultured on human plasma fibronectin coated plates (Millipore; FC010) in N2B27 medium consisting of 48\% DMEM F-12 medium (Invitrogen, 21331-046), 48\% Neurobasal medium (Invitrogen, 21103049), 1\% B27 Supplement w/o vitamin A (Invitrogen, 12587-010), $10 \mathrm{U} / \mathrm{ml}$ penicillin $/ 50 \mu \mathrm{g} / \mathrm{ml}$ streptomycin solution (Sigma, P4333), $2 \mathrm{mM}$ L-glutamine (Sigma, G7513), and $0.15 \mathrm{mM} \beta$-mercaptoethanol (Sigma, M3148), supplemented with $1000 \mathrm{U} / \mathrm{ml}$ Lif (home-made), $3 \mu \mathrm{M}$ CHIR99021 (Biomol, 13122) and $0.4 \mu \mathrm{M}$ PD0325901 (Biomol,13014).

ESC grown in ground state culture conditions were converted to EpiLC ${ }^{26}$ by $48 \mathrm{~h}$ culture in N2B27 medium supplemented with 1\% KSR (Knockout Serum Replacement, Gibco, 10828-028), $20 \mathrm{ng} / \mathrm{ml}$ activin A and $12 \mathrm{ng} / \mathrm{ml} \mathrm{Fgf2.}$

TSC $^{73}$ were maintained on MEFs in MEF medium supplemented with $25 \mathrm{ng} / \mathrm{ml}$ Fgf4 (Peprotech, 100-31) and $1 \mu \mathrm{g} / \mathrm{ml}$ Heparin (Sigma, H3393). ESC were converted to TSC-like cells via forced expression of Cdx2-ERT2 construct $^{24}$, following treatment with $0.5 \mu \mathrm{M}$ 4-Hydroxytamoxifen (Sigma, H7904-5MG).

E3 EpiSC ${ }^{74}$ were cultured in medium containing 50\% N2B27 and 50\% MEFconditioned medium, consisting of DMEM F-12 supplemented with $20 \%$ KSR penicillin-streptomycin, $2 \mathrm{mM}$ L-glutamine, $1 \mathrm{x}$ non-essential amino acids and $0.15 \mathrm{mM} \beta$-mercaptoethanol. The EpiSC medium was supplemented with $10 \mathrm{ng} / \mathrm{ml}$ activin A and $5 \mathrm{ng} / \mathrm{ml} \mathrm{Fgf2} \mathrm{before} \mathrm{use.} \mathrm{The} \mathrm{cells} \mathrm{were} \mathrm{split} \mathrm{every} \mathrm{2-3} \mathrm{days} \mathrm{using}$ accutase (Sigma, A6964).
Conventional hiPSC ${ }^{75}$ were cultured on matrigel coated plates in MEFconditioned medium, supplemented with $10 \mathrm{ng} / \mathrm{ml}$ Fgf2. The cells were split every 3 days using TrypLE Express (Gibco, 12604-013).

Naïve hiPSC ${ }^{76}$ were cultured in PGXL medium consisting of 48\% DMEM F-12 medium, Neurobasal (48\%) medim, 1\% B27 Supplement (Invitrogen, 17504044), $10 \mathrm{U} / \mathrm{ml}$ penicillin $/ 50 \mu \mathrm{g} / \mathrm{ml}$ streptomycin solution, $2 \mathrm{mM} \mathrm{L}$-glutamine and $0.15 \mathrm{mM} \beta$-mercaptoethanol, supplemented with $1 \mu \mathrm{M}$ PD0325901, $2 \mu \mathrm{M}$ Gö6983 (Tebu-bio, 800088), $2 \mu \mathrm{M}$ XAV939 (Sigma, X3004) and $250 \mathrm{U} / \mathrm{ml}$ Lif. The cells were split every 3-4 days using Accutase and cultured on geltrex coated plates (Thermo Fischer Scientific, A1413302).

Xfect (Takara, 631320) or Lipofectamine 2000 (Invitrogen, 11668027) were used for transfection, following the manufacturer's instructions.

Generation of Lima1 KO ESC via CRIPSR/Cas9-mediated deletion. Guide RNAs (guide RNA 1 5'-3': CTGCTCACTTTGTCCTTATA, guide RNA 2 5'-3': GTCGCATGTTAGCTGCAAGA) were designed using the MIT tool (www.crispr.mit.edu). Each of the guide RNA coding sequences was synthesised as a pair of complementary oligonucleotides, which were annealed and separately cloned into pSpCas9(BB)-2A-Puro (PX459) vectors ${ }^{77}$. The vectors were additionally modified to express fluorescent reporters (GFP or Cerulean) in order to facilitate the sorting and collection of double-positive cells. After re-seeding, individual ESC clones were manually picked and expanded. Homozygous deletion of Limal was confirmed by PCR genotyping (primer pair 1 5'-3':

TCTTGTTGTTTGTGGCATAC and CACTCACTTTCCTAACATTGA $-653 \mathrm{bp}$ product of Limal KO allele; primer pair 2 5'-3': GTTTTATCACTTCCTGCTTCA and AAAAGACATACTGTCCACACA -796 bp product of WT Limal locus), as well as by sequencing and western blot analysis.

Fluorescence-activated cell sorting (FACS). Adherent cells were dissociated and resuspended in 3\% FCS/PBS solution (FACS buffer). Chimeric E13.5 embryos were mechanically homogenized, and the cells were subsequently dissociated using trypsin. The cell suspension was pelleted by centrifugation and resuspended in FACS buffer. Single viable cells were selected based on FSC- and SSC-gating. The FACS gating plots are presented in Fig. S8. Cell sorting and analysis was performed using FACSAria IIIu and FACSAria Fusion systems, equipped with FACSDiva Software (v8.01). FlowJo software (v10.7.1) was used for data analysis and plotting.

Immunofluorescence labelling and confocal microscopy. Adherent cells were grown on 8-well $\mu$-slide plates (Ibidi, 80826). Non-adherent cells were collected and pelleted by centrifugation. After washing with PBS, the cells were fixed using $4 \%$ PFA for $15 \mathrm{~min}$ at room temperature and then permeabilized using $0.3 \%$ Triton $\mathrm{X}-100 / \mathrm{PBS}$ for $5 \mathrm{~min}$. After washing, the samples were incubated with blocking buffer (3\% BSA/PBS) for at least $30 \mathrm{~min}$, and after that, the primary antibodies diluted in blocking buffer were applied to the sample and incubated overnight at $4{ }^{\circ} \mathrm{C}$. On the next day, the primary antibody solution was removed, the cells were washed and incubated with secondary antibodies diluted in blocking buffer. Nuclei were counterstained with DAPI.

Pre-implantation embryos were fixed using 4\% PFA/PBS for $10 \mathrm{~min}$, whereas egg cylinder stage embryos were fixed for $15 \mathrm{~min}$. The samples were permeabilised using $0.3 \%$ Triton X-100/PBS solution supplemented with $100 \mathrm{mM}$ glycine for 5 min (pre-implantation embryos) or 15 min (post-implantation embryos). After washing with $5 \%$ FCS/PBS, the samples were incubated with primary antibody solution in $10 \%$ FCS/PBS buffer, overnight at $4{ }^{\circ} \mathrm{C}$. On the next day, the embryos were washed and incubated with secondary antibodies, overnight at $4{ }^{\circ} \mathrm{C}$. After that, the samples were washed and mounted on glass-bottom plates in PBS droplets under oil. Imaging was performed using Zeiss LSM780 system equipped with ZEN software (v2.0 and higher), Fiji software (v2.0 and higher) was used for image analysis. The list of primary and secondary antibodies is presented on Table S2.

Generation of teratomas and immunohistochemistry. Scid mice were injected subcutaneously with a $100 \mu \mathrm{l}$ suspension containing 5 million ESC in PBS. Solid tumours were isolated and fixed at $4{ }^{\circ} \mathrm{C}$ overnight in $4 \%$ PFA/PBS. The samples were dehydrated in an ethanol series $(30 \%, 50 \%, 70 \%$ and $100 \%$ in PBS) for $2 \mathrm{~h}$ each, followed by two $10 \mathrm{~min}$ incubations in $100 \%$ xylene, and then transferred to paraffin. Similarly, foetal stage embryos were fixed overnight and dehydrated. Samples embedded into paraffin blocks were sectioned at $7 \mu \mathrm{m}$ using an HM355S microtome (Thermo). Haematoxylin/eosin staining and immunohistochemistry were carried out as previously described ${ }^{78,79}$. Images were acquired using Nikon Eclipse Ti2 system.

Generation of embryoid bodies. ESC differentiation using the EBs assay was performed as previously described ${ }^{80}$. The EBs were harvested and dissociated into single cells for Seahorse analysis 14 days after induction of differentiation.

Quantitative PCR. RNA isolation was performed using RNeasy Mini Kit (Qiagen, 74106) according to the manufacturer's instructions and reverse transcribed using MMLV-Reverse transcriptase (Applied Biosystems). Transcript levels were quantified using iTaq SYBR Green Supermix (Bio-Rad). Relative gene expression was calculated 
using delta-delta $\mathrm{Ct}$ method and normalized to relative GAPDH expression. Primer pair sequences $\left(5^{\prime}-3^{\prime}\right)$ : Limal (AGCCAGAGACGAGCGAAAAC and GCACCTAT TTCCCAGTCATCCA) Oct4 (AGACCATGTTTCTGAAGTGCCCG and CGCCG GTTACAGAACCATACTCG), Nanog (AGGGTCTGCTACTGAGATGCTCTG and CAACCACTGGTTTTTCTGCCACCG), GAPDH (TGAAGCAGGCATCTGAGGG and CGAAGGTGGAAGAGTGGGAG).

RNA sequencing and bioinformatic analysis. RNA was isolated from cells using the NucleoSpin RNA isolation kit following the manufacturer's protocol (Macherery and Nagel). Samples with RNA integrity number (RIN) above 7 were processed further. The mRNA enrichment which was performed using NEBNext Poly(A) mRNA Magnetic Isolation Module and cDNA library was prepared using NEBNext Ultra II Directional RNA Library Prep Kit. Sequencing was performed on the NextSeq 500 system ( 75 cycles, high output, v2.5) in the Core Facility Genomics of the Medical Faculty, University of Münster.

Reads were trimmed with fastp (v0.20.0), setting the '-3', '-cut_tail_window_size 1 ', and '-cut_tail_mean_quality 20' options. The trimmed RNA-seq data was analyzed with Salmon (v1.4.0) ${ }^{81}$ and DESeq2 (v1.30.0) ${ }^{82}$. Briefly, transcript level abundance for all samples was estimated using Salmon, with indices for the Mus musculus (Gencode v23) and Homo sapiens (Gencode v35) transcriptomes and options '-validateMappings', '-seqBias' and '-gcBias'. The resulting quantification data were imported into R (v4.0.3) with tximeta (v1.8.2) $)^{83}$ and summed up to the gene level. Differential expression per cell type between control/wild-type and Lima1 overexpression/knockdown was tested with DESeq2. Genes with an adjusted $p$-level $<0.01$ were called as differentially expressed. Abundance levels for genes of special interest were z-score transformed and plotted with ComplexHeatmap (v2.6.2) ${ }^{84}$. GO gene set over-representation analysis was performed with clusterProfiler (v3.18.0) $)^{85}$, using the set of all genes with valid GO annotation as background, respectively. All non-heatmap plots were created using ggplot2 (v3.3.2).

ChIP-seq and ATAC-seq data analysis. ChIP-seq dataset of Nanog ${ }^{35}$, Sox $2^{32}$, $\mathrm{Sall}^{33}, \mathrm{NrOb}^{28}{ }^{28} \mathrm{Klf}^{29}{ }^{29}$ Oct $4^{27}, \mathrm{Tcf}^{35}, \beta-\mathrm{cat}^{34}$ and Polr2a ${ }^{31}$ binding in ESC, as well as ESC ATAC-seq data ${ }^{30}$ were obtained from the Cistrome Data Browser ${ }^{86,87}$. Peak visualisation was performed using Integrated Genome Viewer software (v2.4.15).

Protein extraction and immunoblotting. Cells were washed with PBS, scraped and pelleted by centrifugation at $16,000 \times g$ for $10 \mathrm{~min}$. For immunoblotting, the cell pellets were lysed in buffer consisting of $10 \mathrm{mM}$ Tris- $\mathrm{HCl} \mathrm{pH} \mathrm{7.6,150 \textrm {mM }}$ $\mathrm{NaCl}, 2 \mathrm{mM} \mathrm{MgCl}$, $2 \mathrm{mM}$ EDTA, $0.1 \%$ Triton X-100, 10\% Glycerol and protease inhibitors (Sigma, 5892791001) for $15 \mathrm{~min}$ on ice. For mass spectrometry analysis, the cells were lysed in buffer consisting of $8 \mathrm{M}$ Urea, $50 \mathrm{mM}$ Tris- $\mathrm{HCl} \mathrm{pH} 8.0$, $150 \mathrm{mM} \mathrm{NaCl}, 1 \mathrm{mM}$ EDTA and protease inhibitors for $15 \mathrm{~min}$ on ice. After that supernatants were collected by centrifugation at $16,000 \times g$ for $15 \mathrm{~min}$ and the protein concentration was determined using BCA kit (Thermo, 23227) following manufacturer's instructions. The protein samples were run on SDS-Polyacrylamide gel alongside and then transferred onto a PVDF membrane. After that the membrane was incubated in 5\% dry milk or BSA in PBST (0.01\% Tween-20/PBS) buffer, for $30 \mathrm{~min}$ at room temperature and then incubated with primary antibodies at $4{ }^{\circ} \mathrm{C}$, overnight. On the next day, the membrane was washed with PBST and incubated with HRP-conjugated secondary antibodies for $45 \mathrm{~min}$ at room temperature. The proteins were detected using the ECL (GE Healthcare, GERPN3243) or ECL Prime (GE Healthcare, GERPN2232) kits by exposing the membranes to ECL Hyperfilms (GE Healthcare, GE28-9068-36). Scans of the immunoblots used in this study are presented in Fig. S9.

Proximity biotinylation. APEX2 proximity biotinylation in ESC expressing fulllength Lima1-HA-APEX2 construct was performed following previously published protocol $^{88}$. Briefly, the cells were cultured in the presence of $500 \mu \mathrm{M}$ biotin-phenol for $30 \mathrm{~min}$, followed by pulse treatment with $1 \mathrm{mM} \mathrm{H}_{2} \mathrm{O}_{2}$ for $1 \mathrm{~min}$. After extensive washing with quenching buffer $(10 \mathrm{mM}$ sodium ascorbate, $5 \mathrm{mM}$ Trolox, $10 \mathrm{mM}$ sodium azide in PBS), the cells were lysed and biotinylation was verified by immunoblotting (see Protein extraction and Immunoblotting) using the following modifications in the protocol: After transfer to PDVF membrane, the membrane was blocked with 5\% skim milk in $0.2 \%$ Triton X-100/TBS (TBS-Triton) for $1 \mathrm{~h}$, washed briefly three times with $0.05 \%$ Tween- 20 in TBS (TBS-Tween) and incubated with streptavidin-HRP in 3\% BSA in TBS-Triton for $1 \mathrm{~h}$. Following extensive washing (three times in TBS-Tween, one time in 10\% FCS/ 1\% Triton X-100/TBSTween and five times in TBS-Tween), protein biotinylation was visualized using ECL kit, by exposing the membranes to ECL Hyperfilms.

Approximately $1 \mathrm{mg}$ of protein lysate was pre-digested using endoproteinase LysC $(1: 100, w / w)$ for $2 \mathrm{~h}$ at $30^{\circ} \mathrm{C}$. Next, the concentration of urea was reduced to $2 \mathrm{M}$ using $50 \mathrm{mM}$ ammonium bicarbonate buffer $(\mathrm{pH} \mathrm{8.0)}$ ) and the digestion was extended overnight at $37^{\circ} \mathrm{C}$ by addition of trypsin $(1: 50, \mathrm{w} / \mathrm{w})$. The digestion was stopped by the addition of trifluoroacetic acid (TFA) to a final concentration of $1 \%$, desalted using C18 Sep-Pak cartridges (Waters) and lyophilized. Lyophilizates were dissolved in $1.5 \mathrm{ml}$ IAP buffer $(10 \mathrm{mM}$ sodium phosphate dibasic, $50 \mathrm{mM}$ MOPS $\mathrm{pH} 7.2,50 \mathrm{mM} \mathrm{NaCl}$ ). The $\mathrm{pH}$ was adjusted to seven with $1 \mathrm{M}$ Tris. The pull-down was performed following previously published protocol ${ }^{89}$. In brief, $20 \mu$ of agarose beads covalently linked to biotin antibody (ImmuneChem, ICP0615) were first washed with IAP buffer. After that, the beads were incubated with the sample at $4{ }^{\circ} \mathrm{C}$ for $1 \mathrm{~h}$ on a rotator. Following four times washing with PBS, the bound peptides were eluted using $0.15 \%(\mathrm{v} / \mathrm{v}) \mathrm{TFA}$, desalted using C18 Stage tips and stored on-tip until subjected to measurement on the MS system.

Mass spectrometry. Peptide samples in $0.1 \%$ formic acid were measured on a hybrid TIMS-quadrupole time of flight mass spectrometer (timsTOF pro) coupled to a nanoElute UHPLC system via a Captive Spray ion source (Bruker). Peptides were separated on the nanoElute within 90 min with a linear gradient from 3-35\% buffer B on a self-packed C18 reverse phase capillary column with pulled emitter tip (nanoseparations; $360 \mu \mathrm{m}$ OD x $75 \mu \mathrm{m}$ ID $\times 250 \mathrm{~mm}$; Reprosil pur C18-aq, $1.9 \mu \mathrm{m}$; Dr Maisch) using a constant flow of $300 \mathrm{nl} / \mathrm{min}$ (Buffer A: $0.1 \%$ formic acid; Buffer B: $0.1 \%$ formic acid in acetonitrile). At the end of the gradient, the column was flushed with $90 \%$ B before re-equilibration at starting conditions. MS and MS/MS spectra were recorded in positive mode from m/z 100 to $1700 \mathrm{Da}$, using the PASEF scan mode. Each duty cycle consisted of 1 TIMS-MS and an average of ten PASEF MS/MS frames, each one containing multiple MS/MS spectra, which resulted in a total cycle time of $1.1 \mathrm{~s}$. To exclude the majority of singly charged ions with low $\mathrm{m} / \mathrm{z}$ for PASEF precursor selection, a polygon filtering was applied to the $\mathrm{m} / \mathrm{z}$ over ion mobility area. For the $90 \mathrm{~min}$ runs target intensity was set to $20,000 \mathrm{cts} / \mathrm{s}$ and an ion mobility range $(1 / \mathrm{K} 0)$ of $0.6-1.6 \mathrm{Vs} / \mathrm{cm}^{2}$ was used. Data were acquired with a $100 \mathrm{~ms}$ ramp time. The Bruker Hystar/oTOF Control software was used to control the LC-MS system and record the data (version 3.2; Bruker Daltonics).

Mass spectrometry data analysis and label-free quantification. MS files were processed using the MaxQuant computational platform (version 1.6.14.0) ${ }^{90}$. Identification of peptides and proteins was enabled by the built-in Andromeda search engine by querying the concatenated forward and reverse mouse Uniprot database (UP000005640_9606.fasta; version April 2019) including common lab contaminants. Default values of MaxQuant remained unchanged. Trypsin was selected as a protease allowing up to two missed cleavages, and the peptide search was limited to a minimum length of 7 amino acids and a maximum mass of $4600 \mathrm{Da}$. Oxidation of methionine, protein N-terminal acetylation, deamidation as well as the biotinylation of lysine $(+226.07759 \mathrm{Da})$, biotin-phenol modification of tyrosine $(+361.14601 \mathrm{Da})$, and oxidized-biotin-phenol modification of tyrosine $(+377.141 \mathrm{Da})$ were set as variable modifications, while carbamidomethylations of cysteine were defined as fixed modification. For peptide and protein identifications, a minimum false discovery rate (FDR) of $1 \%$ was required. The match between runs option was enabled setting a retention time matching window of $0.7 \mathrm{~min}$ that included also a $1 / \mathrm{K} 0$ matching window of $0.05 \mathrm{~V} \cdot \mathrm{s} / \mathrm{cm}^{2}$ for measurements that were obtained on the timsTOF. The calculation of iBAQ values was enabled on all occasions.

Relative label-free quantification using the MaxQuant LFQ algorithm (version 1.6.14.0) was based on the measurements of three biological replicates for each sample. First, reverse and contaminant hits as well as proteins that were identified by a single modified peptide only were eliminated from the list of identified protein groups. Proteins eventually included for further analysis had to be identified with at least one unique peptide.

Following the labelling reaction and subsequent sample preparation steps, biotin-phenol containing peptides were specifically enriched. Matching back those labelled peptides to the proteins from which they were derived, indicated that these proteins must have been present in close proximity to the APEX enzyme. In contrast, control samples that were incubated without the biotin-phenol substrate were completely void of those modified peptides. Thus, while we formally cannot exclude the possibility that also more abundant proteins were accidentally present in the vicinity of Limal during the short labelling reaction and could have been labelled by the APEX activity, we consider that primarily static, as well as more dynamically binding interactors of Limal, were predominantly labelled.

TMRE assay. The cells were incubated in culture medium supplemented with $300 \mathrm{nM}$ MitoTracker Green (Invitrogen, M7514) for $30 \mathrm{~min}$. After that the cells were washed twice with PBS and once with culture medium and then incubated in medium supplemented with $15 \mathrm{nM}$ TMRE (Biomol, ABD-22220) for $30 \mathrm{~min}$. The negative controls were incubated additionally with $20 \mu \mathrm{M}$ of the oxidative phosphorylation uncoupler carbonyl cyanide m-chlorophenylhydrazone (CCCP; Sigma, C2759) for $10 \mathrm{~min}$. After that cells were washed, harvested and subjected to FACS analysis. DAPI was used to select the live cells. The TMRE signal intensity was normalized to MitoTracker Green signal.

Pyruvate kinase activity assay. Photometric analysis of pyruvate kinase activity in ESC lysates was performed using a pyruvate kinase activity kit (Sigma, MAK072), following the manufacturer's instruction.

ROS assay. Analysis of ROS levels was performed using ROS detection assay kit (PromoCell, PK-CA577-K936-1) according to the manufacturer's instructions. 
Mitochondrial stress test assay. The OCR was measured with the Seahorse XFe96 Extracellular Flux Analyzer. The cells were seeded on 96-well XF cell culture microplates $24 \mathrm{~h}$ prior to analysis. Fresh medium was supplied before starting the OCR analysis. The assay was performed according to the manufacturer's instructions using $0.5 \mu \mathrm{M}$ Oligomycin, $1 \mu \mathrm{M}$ FCCP and $0.5 \mu \mathrm{M}$ Rotenone/Antimycin A. After the OCR measurement, the cell number per well was determined by labelling the nuclei with Hoechst 33342.

Annexin V assay. ESCs were individualized by trypsinization, whereas hiPSCs were individualized by Accutase treatment for $10 \mathrm{~min}$ at $37^{\circ} \mathrm{C}$. The cells were then resuspended and cultured on cell-repellent plates for $2 \mathrm{~h}$ at $37^{\circ} \mathrm{C}$. After that, the cell death was determined by FACS analysis using Annexin V Alexa Fluor 568 conjugate kit (Thermo, A13202), according to the manufacturer's instructions. DAPI was added just before the FACS analysis.

Ultrastructural analysis. Cells were initially fixed in $2 \%$ paraformaldehyde, $0.2 \%$ glutaraldehyde in $0.1 \mathrm{M}$ phosphate buffer, $\mathrm{pH} 7.2$, and further processed for cryo immunogold labelling as previously described ${ }^{91}$. From the resulting frozen sample blocks, $50 \mathrm{~nm}$ ultrathin sections were cut at $-110^{\circ} \mathrm{C}$. The sections were retrieved in a mixture of sucrose/methylcellulose and transferred on formvar coated copper grids (200-mesh, hexagonal). Single immunogold labelling was performed against HA-tagged Limal with an antibody recognizing HA-tag (mouse, clone 16.B12, Biolegend), detected by a bridging antibody, which was recognized by $10 \mathrm{~nm}$ protein A gold conjugate (CMC, Utrecht, Netherlands).

For double immunogold labelling antibodies against actin (mouse, 4F7, kind gift from Professor Brigitte Jockusch and Sabine Buchmeier, Antibody Facility TUBraunschweig) and Limal (rabbit, A300-102A, Bethyl Laboratories, Inc.) were used stepwise, detected by $15 \mathrm{~nm}$ and $10 \mathrm{~nm}$ protein A gold, respectively. The samples were analyzed at $80 \mathrm{kV}$ using a transmission electron microscope (Tecani12Biotwin, Thermo Fisher Scientific). Representative images were exposed on ditabis imaging plates (Ditabis, Pforzheim, Germany).

Quantification and statistical analysis. All statistical analyses were performed using GraphPad Prism unless otherwise stated. Information about number of independent repetitions, sample size and statistical tests are indicated in the figure legends.

Reporting summary. Further information on research design is available in the Nature Research Reporting Summary linked to this article.

\section{Data availability}

The RNA-seq data generated in this study have been deposited in the ArrayExpress database under accession code "E-MTAB-10301". The proteomics data data data generated in this study have been deposited in the ProteomeXchange database under accession code “ PXSD025293”. Uniprot database UP000005640_9606.fasta; version April 2019 used for Mass Spectrometry analysis is available at "Uniprot [ftp:// ftp.uniprot.org/pub/databases/uniprot/previous_releases/release-2019_04/

knowledgebase/]". All other relevant data supporting the key findings of this study are available within the article and its Supplementary Information files or from the corresponding author upon reasonable request. Source data are provided with this paper.

Received: 5 May 2021; Accepted: 10 January 2022;

Published online: 01 February 2022

\section{References}

1. Stemmler, M. P. Cadherins in development and cancer. Mol Biosyst 4, 835-850 (2008).

2. Hanahan, D. \& Weinberg, R. A. Hallmarks of cancer: the next generation. Cell 144, 646-674 (2011).

3. Aiello, N. M. \& Stanger, B. Z. Echoes of the embryo: using the developmental biology toolkit to study cancer. Dis Model Mech 9, 105-114 (2016).

4. Fan, R. et al. Wnt/Beta-catenin/Esrrb signalling controls the tissue-scale reorganization and maintenance of the pluripotent lineage during murine embryonic diapause. Nat Commun 11, 5499 (2020).

5. Steinhart, Z. \& Angers, S. Wnt signaling in development and tissue homeostasis. Development https://doi.org/10.1242/dev.146589 (2018).

6. Zhan, T., Rindtorff, N. \& Boutros, M. Wnt signaling in cancer. Oncogene 36, 1461-1473 (2017).

7. Abe, K. \& Takeichi, M. EPLIN mediates linkage of the cadherin catenin complex to F-actin and stabilizes the circumferential actin belt. Proc. Natl Acad Sci. USA 105, 13-19 (2008).

8. Collins, R. J., Jiang, W. G., Hargest, R., Mason, M. D. \& Sanders, A. J. EPLIN: A fundamental actin regulator in cancer metastasis? Cancer Metastasis Rev. 34, 753-764 (2015)
9. Chen, S., Maul, R. S., Kim, H. R. \& Chang, D. D. Characterization of the human EPLIN (Epithelial Protein Lost in Neoplasm) gene reveals distinct promoters for the two EPLIN isoforms. Gene 248, 69-76 (2000).

10. Maul, R. S. \& Chang, D. D. EPLIN, epithelial protein lost in neoplasm. Oncogene 18, 7838-7841 (1999).

11. Maul, R. S. et al. EPLIN regulates actin dynamics by cross-linking and stabilizing filaments. J Cell Biol. 160, 399-407 (2003).

12. Buckley, C. D. et al. Cell adhesion. The minimal cadherin-catenin complex binds to actin filaments under force. Science 346, 1254211 (2014)

13. Taguchi, K., Ishiuchi, T. \& Takeichi, M. Mechanosensitive EPLIN-dependent remodeling of adherens junctions regulates epithelial reshaping. J Cell Biol. 194, 643-656 (2011).

14. Chang, D. D., Park, N. H., Denny, C. T., Nelson, S. F. \& Pe, M. Characterization of transformation related genes in oral cancer cells. Oncogene 16, 1921-1930 (1998).

15. Collins, R. J. et al. Mechanistic insights of epithelial protein lost in neoplasm in prostate cancer metastasis. Int J Cancer 143, 2537-2550 (2018).

16. Liu, Y., Sanders, A. J., Zhang, L. \& Jiang, W. G. EPLIN-alpha expression in human oesophageal cancer and its impact on cellular aggressiveness and clinical outcome. Anticancer Res. 32, 1283-1289 (2012).

17. Jiang, W. G. et al. Eplin-alpha expression in human breast cancer, the impact on cellular migration and clinical outcome. Mol Cancer 7, 71 (2008).

18. Zhang, S. et al. EPLIN downregulation promotes epithelial-mesenchymal transition in prostate cancer cells and correlates with clinical lymph node metastasis. Oncogene 30, 4941-4952 (2011).

19. Zhang, Y. Y. et al. A LIMAl variant promotes low plasma LDL cholesterol and decreases intestinal cholesterol absorption. Science 360, 1087-1092 (2018).

20. Wang, Q. T. et al. A genome-wide study of gene activity reveals developmental signaling pathways in the preimplantation mouse embryo. Dev Cell 6, 133-144 (2004).

21. Williams, R. L. et al. Myeloid leukaemia inhibitory factor maintains the developmental potential of embryonic stem cells. Nature 336, 684-687 (1988)

22. Ying, Q. L. et al. The ground state of embryonic stem cell self-renewal. Nature 453, 519-523 (2008).

23. Tanaka, S., Kunath, T., Hadjantonakis, A. K., Nagy, A. \& Rossant, J. Promotion of trophoblast stem cell proliferation by FGF4. Science 282, 2072-2075 (1998)

24. Niwa, $\mathrm{H}$. et al. Interaction between Oct $3 / 4$ and $\mathrm{Cdx} 2$ determines trophectoderm differentiation. Cell 123, 917-929 (2005).

25. Boroviak, T., Loos, R., Bertone, P., Smith, A. \& Nichols, J. The ability of innercell-mass cells to self-renew as embryonic stem cells is acquired following epiblast specification. Nature Cell Bio. 16, $513+(2014)$

26. Hayashi, K., Ohta, H., Kurimoto, K., Aramaki, S. \& Saitou, M. Reconstitution of the mouse germ cell specification pathway in culture by pluripotent stem cells. Cell 146, 519-532 (2011).

27. Buecker, C. et al. Reorganization of enhancer patterns in transition from naive to primed pluripotency. Cell Stem Cell 14, 838-853 (2014).

28. Beck, S. et al. CpG island-mediated global gene regulatory modes in mouse embryonic stem cells. Nat Commun 5, 5490 (2014).

29. Liu, Y. et al. Widespread mitotic bookmarking by histone marks and transcription factors in pluripotent stem cells. Cell Rep 19, 1283-1293 (2017)

30. Hodges, H. C. et al. Dominant-negative SMARCA4 mutants alter the accessibility landscape of tissue-unrestricted enhancers. Nat Struct Mol Biol. 25, 61-72 (2018).

31. Dorighi, K. M. et al. Mll3 and Mll4 facilitate enhancer RNA synthesis and transcription from promoters independently of H3K4 monomethylation. $\mathrm{Mol}$ Cell 66, 568-576.e4 (2017).

32. Liu, Z. \& Kraus, W. L. Catalytic-independent functions of PARP-1 determine Sox2 pioneer activity at intractable genomic loci. Mol Cell 65, 589-603.e9 (2017).

33. Xiong, J. et al. Cooperative action between SALL4A and TET proteins in stepwise oxidation of 5-methylcytosine. Mol Cell 64, 913-925 (2016).

34. Zhang, X., Peterson, K. A., Liu, X. S., McMahon, A. P. \& Ohba, S. Gene regulatory networks mediating canonical Wnt signal-directed control of pluripotency and differentiation in embryo stem cells. Stem Cells 31, 2667-2679 (2013).

35. Marson, A. et al. Connecting microRNA genes to the core transcriptional regulatory circuitry of embryonic stem cells. Cell 134, 521-533 (2008).

36. Hung, V. et al. Spatially resolved proteomic mapping in living cells with the engineered peroxidase APEX2. Nat Protoc 11, 456-475 (2016)

37. Taha, M. et al. EPLIN-alpha and -beta isoforms modulate endothelial cell dynamics through a spatiotemporally differentiated interaction with actin. Cell Rep 29, 1010-1026 (2019). e1016.

38. Charras, G. \& Paluch, E. Blebs lead the way: how to migrate without lamellipodia. Nat. Rev. Mol. Cell Bio. 9, 730-736 (2008).

39. Cunningham, C. C. Actin polymerization and intracellular solvent flow in cell surface blebbing. J Cell Biol. 129, 1589-1599 (1995).

40. Paluch, E., Piel, M., Prost, J., Bornens, M. \& Sykes, C. Cortical actomyosin breakage triggers shape oscillations in cells and cell fragments. Biophys $J \mathbf{8 9}$, 724-733 (2005). 
41. Keller, H. \& Eggli, P. Protrusive activity, cytoplasmic compartmentalization, and restriction rings in locomoting blebbing Walker carcinosarcoma cells are related to detachment of cortical actin from the plasma membrane. Cell Motil Cytoskeleton 41, 181-193 (1998).

42. Ohgushi, M. et al. Molecular pathway and cell state responsible for dissociation-induced apoptosis in human pluripotent stem cells. Cell Stem Cell 7, 225-239 (2010).

43. Watanabe, K. et al. A ROCK inhibitor permits survival of dissociated human embryonic stem cells. Nat Biotechnol 25, 681-686 (2007).

44. Evans, M. J. \& Kaufman, M. H. Establishment in culture of pluripotential cells from mouse embryos. Nature 292, 154-156 (1981)

45. Tanimura, N. \& Fujita, Y. Epithelial defense against cancer (EDAC). Semin Cancer Biol. 63, 44-48 (2020).

46. Ohoka, A. et al. EPLIN is a crucial regulator for extrusion of RasV12transformed cells. J Cell Sci. 128, 781-789 (2015).

47. Kon, S. et al. Cell competition with normal epithelial cells promotes apical extrusion of transformed cells through metabolic changes. Nat Cell Biol. 19, 530-541 (2017).

48. Mitchell, P. Coupling of phosphorylation to electron and hydrogen transfer by a chemi-osmotic type of mechanism. Nature 191, 144-148 (1961).

49. Zhou, W. et al. HIFlalpha induced switch from bivalent to exclusively glycolytic metabolism during ESC-to-EpiSC/hESC transition. EMBO J 31, 2103-2116 (2012).

50. Giulitti, S. et al. Direct generation of human naive induced pluripotent stem cells from somatic cells in microfluidics. Nat Cell Biol. 21, 275-286 (2019).

51. Namgoong, S. \& Kim, N. H. Roles of actin binding proteins in mammalian oocyte maturation and beyond. Cell Cycle 15, 1830-1843 (2016).

52. Leader, B. et al. Formin-2, polyploidy, hypofertility and positioning of the meiotic spindle in mouse oocytes. Nat Cell Biol. 4, 921-928 (2002).

53. Yi, K. et al. Dynamic maintenance of asymmetric meiotic spindle position through Arp2/3-complex-driven cytoplasmic streaming in mouse oocytes. Nat Cell Biol. 13, 1252-1258 (2011).

54. Chircop, M. et al. The actin-binding and bundling protein, EPLIN, is required for cytokinesis. Cell Cycle 8, 757-764 (2009).

55. Govindasamy, N., Duethorn, B., Oezgueldez, H. O., Kim, Y. S. \& Bedzhov, I. Test-tube embryos-mouse and human development in vitro to blastocyst stage and beyond. Int J Dev Biol. 63, 203-215 (2019).

56. Yanagida, A. et al. Cell surface fluctuations regulate early embryonic lineage sorting. bioRxiv https://doi.org/10.1101/2020.08.16.250084 (2020).

57. Mathieu, J. \& Ruohola-Baker, H. Metabolic remodeling during the loss and acquisition of pluripotency. Development 144, 541-551 (2017).

58. Sperber, H. et al. The metabolome regulates the epigenetic landscape during naive-to-primed human embryonic stem cell transition. Nat Cell Biol. 17, 1523-1535 (2015).

59. Takashima, Y. et al. Resetting transcription factor control circuitry toward ground-state pluripotency in human. Cell 158, 1254-1269 (2014).

60. Lima, A. et al. Differences in mitochondrial activity trigger cell competition during early mouse development. bioRxiv, 2020.2001.2015.900613, https:// doi.org/10.1101/2020.01.15.900613 (2020).

61. Lakso, M. et al. Efficient in vivo manipulation of mouse genomic sequences at the zygote stage. Proc Natl Acad Sci. USA 93, 5860-5865 (1996).

62. $\mathrm{Hu}, \mathrm{H}$. et al. Phosphoinositide 3-kinase regulates glycolysis through mobilization of aldolase from the actin cytoskeleton. Cell 164, 433-446 (2016).

63. Bays, J. L., Campbell, H. K., Heidema, C., Sebbagh, M. \& DeMali, K. A. Linking E-cadherin mechanotransduction to cell metabolism through forcemediated activation of AMPK. Nat Cell Biol. 19, 724-731 (2017).

64. Chervin-Petinot, A. et al. Epithelial protein lost in neoplasm (EPLIN) interacts with alpha-catenin and actin filaments in endothelial cells and stabilizes vascular capillary network in vitro. J Biol. Chem. 287, 7556-7572 (2012).

65. Han, M. Y., Kosako, H., Watanabe, T. \& Hattori, S. Extracellular signalregulated kinase/mitogen-activated protein kinase regulates actin organization and cell motility by phosphorylating the actin cross-linking protein EPLIN. Mol. Cell Biol. 27, 8190-8204 (2007).

66. Ohtsuka, S., Nishikawa-Torikai, S. \& Niwa, H. E-cadherin promotes incorporation of mouse epiblast stem cells into normal development. PLoS One 7, e45220 (2012).

67. Huang, K. et al. BMI1 enables interspecies chimerism with human pluripotent stem cells. Nat Commun 9, 4649 (2018).

68. Masaki, H. et al. Inhibition of apoptosis overcomes stage-related compatibility barriers to chimera formation in mouse embryos. Cell Stem Cell 19, 587-592 (2016).

69. Zheng, C. et al. Cell competition constitutes a barrier for interspecies chimerism. Nature, https://doi.org/10.1038/s41586-021-03273-0 (2021).

70. Wolf, K. et al. Compensation mechanism in tumor cell migration: mesenchymal-amoeboid transition after blocking of pericellular proteolysis. $J$ Cell Biol. 160, 267-277 (2003).

71. Friedl, P. \& Wolf, K. Tumour-cell invasion and migration: diversity and escape mechanisms. Nat Rev Cancer 3, 362-374 (2003).
72. Ozguldez, H. O., Fan, R. \& Bedzhov, I. Placental gene editing via trophectoderm-specific Tat-Cre/loxP recombination. Development https:// doi.org/10.1242/dev.190371 (2020).

73. Kubaczka, C. et al. Derivation and maintenance of murine trophoblast stem cells under defined conditions. Stem Cell Reports 2, 232-242 (2014).

74. Han, D. W. et al. Epiblast stem cell subpopulations represent mouse embryos of distinct pregastrulation stages. Cell 143, 617-627 (2010).

75. Kim, K. P. et al. Reprogramming competence of OCT factors is determined by transactivation domains. Sci. Adv https://doi.org/10.1126/sciadv.aaz7364 (2020).

76. Guo, G. et al. Epigenetic resetting of human pluripotency. Development 144, 2748-2763 (2017)

77. Ran, F. A. et al. Genome engineering using the CRISPR-Cas9 system. Nat. Protoc 8, 2281-2308 (2013).

78. Stemmler, M. P. \& Bedzhov, I. A Cdh1HA knock-in allele rescues the Cdh1-/phenotype but shows essential Cdh1 function during placentation. Dev Dyn 239, 2330-2344 (2010).

79. Bedzhov, I., Liszewska, E., Kanzler, B. \& Stemmler, M. P. Igf1r signaling is indispensable for preimplantation development and is activated via a novel function of E-cadherin. PLoS Genet 8, e1002609 (2012).

80. Bedzhov, I. et al. Adhesion, but not a specific cadherin code, is indispensable for ES cell and induced pluripotency. Stem Cell Res. 11, 1250-1263 (2013).

81. Patro, R., Duggal, G., Love, M. I., Irizarry, R. A. \& Kingsford, C. Salmon provides fast and bias-aware quantification of transcript expression. Nat Methods 14, 417-419 (2017).

82. Love, M. I., Huber, W. \& Anders, S. Moderated estimation of fold change and dispersion for RNA-seq data with DESeq2. Genome Biol. 15, 550 (2014).

83. Love, M. I. et al. Tximeta: Reference sequence checksums for provenance identification in RNA-seq. PLoS Comput Biol. 16, el007664 (2020).

84. Gu, Z., Eils, R. \& Schlesner, M. Complex heatmaps reveal patterns and correlations in multidimensional genomic data. Bioinformatics 32, 2847-2849 (2016).

85. Yu, G., Wang, L. G., Han, Y. \& He, Q. Y. clusterProfiler: an R package for comparing biological themes among gene clusters. OMICS 16, 284-287 (2012).

86. Mei, S. et al. Cistrome data browser: a data portal for ChIP-Seq and chromatin accessibility data in human and mouse. Nucleic Acids Res. 45, D658-D662 (2017).

87. Zheng, R. et al. Cistrome data browser: expanded datasets and new tools for gene regulatory analysis. Nucleic Acids Res. 47, D729-D735 (2019).

88. Han, S. et al. Proximity biotinylation as a method for mapping proteins associated with mtDNA in living cells. Cell Chem Biol. 24, 404-414 (2017).

89. Udeshi, N. D. et al. Antibodies to biotin enable large-scale detection of biotinylation sites on proteins. Nat Methods 14, 1167-1170 (2017).

90. Cox, J. \& Mann, M. MaxQuant enables high peptide identification rates, individualized p.p.b.-range mass accuracies and proteome-wide protein quantification. Nat Biotechnol 26, 1367-1372 (2008).

91. Slot, J. W. \& Geuze, H. J. Cryosectioning and immunolabeling. Nat Protoc 2, 2480-2491 (2007)

\section{Acknowledgements}

We thank Prof. Dr Rolf Kemler for the fruitful discussions and continuous support; Prof Hans R. Schöler, Prof. Dr Ralf H. Adams and Prof. Dr Dietmar Vestweber (MPI-MB) for providing access to key infrastructure, equipment and reagents; Prof. Dr Roland Wedlich-Söldner (University of Münster) for sharing reagents; Prof. Dr Masatoshi Takeichi (RIKEN Center for Developmental Biology) for providing Limal antibody and plasmids; Dr Anika Witten for the RNA sequencing, Prof. Hubert Schorle (University of Bonn) for sharing TSC, Kirill Salewskij and all members of the Embryonic SelfOrganization research group and the Department of Cell and Developmental Biology (MPI-MB) for the fruitful discussions and suggestions and Dr Celeste Brennecka for proofreading the manuscript. This work was supported by the German Research Foundation (DFG) grant BE-5800/2-1 and DFG Emmy Noether grant BE-5800/1-1 to I.B.; Wellcome Trust $(098287 / Z / 12 / Z)$ and ERC (669198) grants to M.Z.-G.; B.D. is a member of and was supported by the CiM/IMPRS joint Graduate School.

\section{Author contributions}

I.B. and B.D. conceived the study and designed the experiments; B.D. performed most of the experiments; F.G. and J.M.V. performed the bioinformatical analysis; B.R., M.-T.B. and K.B.B. performed the Seahorse measurements; H.C.A.D. performed the mass spectrometry; H.B. performed histology; L.K. did the embryo transfers; K.M. and D.Z. performed ultrastructural analysis; M.S. assisted with FACS and FlowJo analysis; M.Z.-G. and M.P.S. provided access to key infrastructure and critical discussion of the manuscript; I.B. and B.D. wrote the manuscript.

\section{Funding}

Open Access funding enabled and organized by Projekt DEAL.

\section{Competing interests}

The authors declare no competing interests. 


\section{Additional information}

Supplementary information The online version contains supplementary material available at https://doi.org/10.1038/s41467-022-28139-5.

Correspondence and requests for materials should be addressed to Ivan Bedzhov.

Peer review information Nature Communications thanks Jiyeon Kim and the other anonymous reviewer(s) for their contribution to the peer review of this work.

Reprints and permission information is available at http://www.nature.com/reprints

Publisher's note Springer Nature remains neutral with regard to jurisdictional claims in published maps and institutional affiliations. (c) (P) Open Access This article is licensed under a Creative Commons Attribution 4.0 International License, which permits use, sharing, adaptation, distribution and reproduction in any medium or format, as long as you give appropriate credit to the original author(s) and the source, provide a link to the Creative Commons license, and indicate if changes were made. The images or other third party material in this article are included in the article's Creative Commons license, unless indicated otherwise in a credit line to the material. If material is not included in the article's Creative Commons license and your intended use is not permitted by statutory regulation or exceeds the permitted use, you will need to obtain permission directly from the copyright holder. To view a copy of this license, visit http://creativecommons.org/ licenses/by/4.0/.

(C) The Author(s) 2022 